\title{
Electric Grid Expansion Planning with High Levels of Variable Generation
}

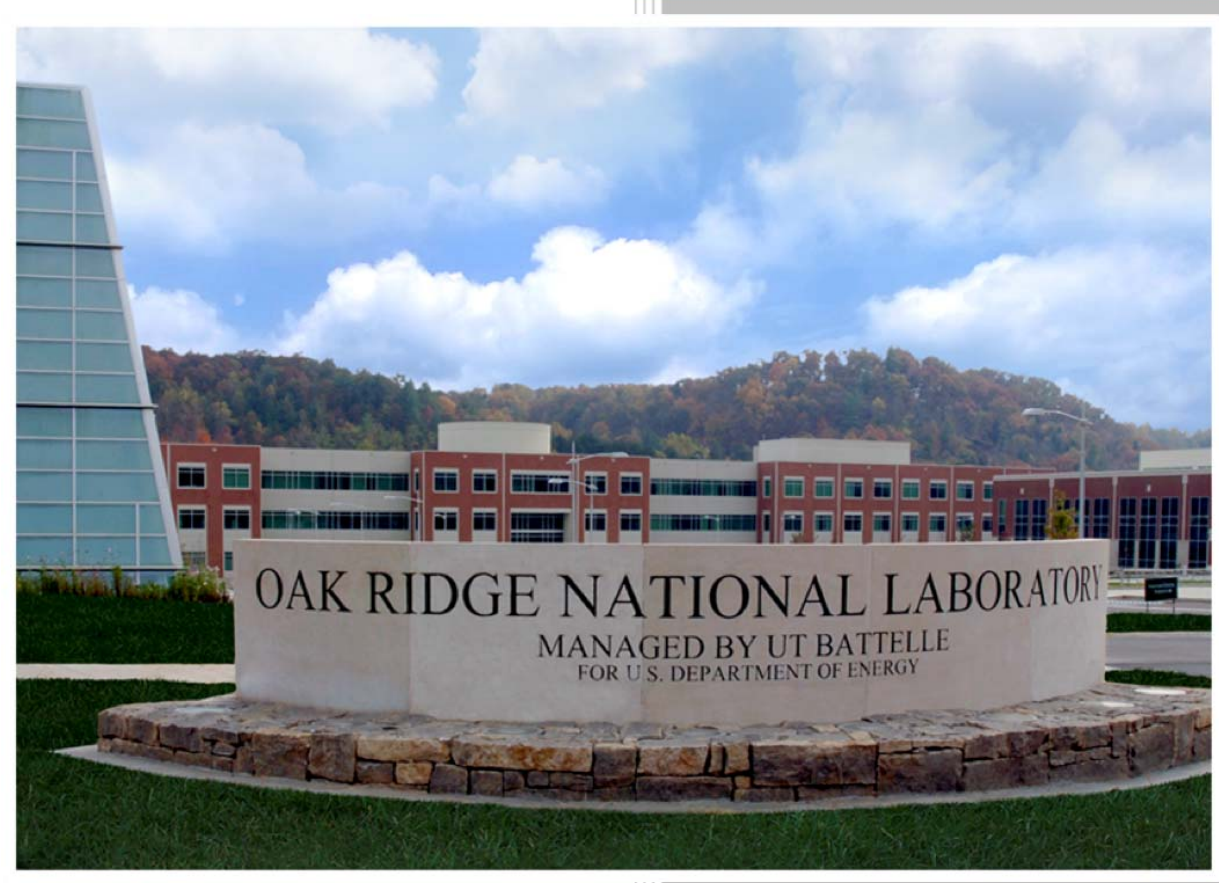

Stanton W. Hadley

Shutang You

Approved for public release.

Distribution is unlimited.

Mallikarjun Shankar

Yilu Liu

February 2016 


\title{
DOCUMENT AVAILABILITY
}

Reports produced after January 1, 1996, are generally available free via US Department of Energy (DOE) SciTech Connect.

Website http://www.osti.gov/scitech/

Reports produced before January 1, 1996, may be purchased by members of the public from the following source:

\author{
National Technical Information Service \\ 5285 Port Royal Road \\ Springfield, VA 22161 \\ Telephone 703-605-6000 (1-800-553-6847) \\ TDD 703-487-4639 \\ Fax 703-605-6900 \\ E-mail info@ntis.gov \\ Website http://www.ntis.gov/help/ordermethods.aspx
}

Reports are available to DOE employees, DOE contractors, Energy Technology Data Exchange representatives, and International Nuclear Information System representatives from the following source:

Office of Scientific and Technical Information

PO Box 62

Oak Ridge, TN 37831

Telephone 865-576-8401

Fax 865-576-5728

E-mail reports@osti.gov

Website http://www.osti.gov/contact.html

This report was prepared as an account of work sponsored by an agency of the United States Government. Neither the United States Government nor any agency thereof, nor any of their employees, makes any warranty, express or implied, or assumes any legal liability or responsibility for the accuracy, completeness, or usefulness of any information, apparatus, product, or process disclosed, or represents that its use would not infringe privately owned rights. Reference herein to any specific commercial product, process, or service by trade name, trademark, manufacturer, or otherwise, does not necessarily constitute or imply its endorsement, recommendation, or favoring by the United States Government or any agency thereof. The views and opinions of authors expressed herein do not necessarily state or reflect those of the United States Government or any agency thereof. 


\title{
ELECTRIC GRID EXPANSION PLANNING WITH HIGH LEVELS OF VARIABLE GENERATION
}

\author{
Stanton W. Hadley \\ Shutang You* \\ Mallikarjun Shankar \\ Yilu Liu*
}

*University of Tennessee - Knoxville

February 2016

Prepared by

OAK RIDGE NATIONAL LABORATORY

Oak Ridge, TN 37831-6283

managed by

UT-BATTELLE, LLC

for the

US DEPARTMENT OF ENERGY

under contract DE-AC05-00OR22725 



\section{CONTENTS}

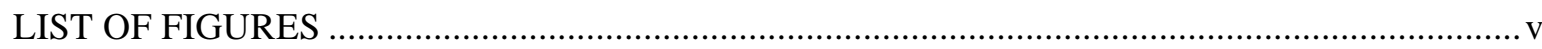

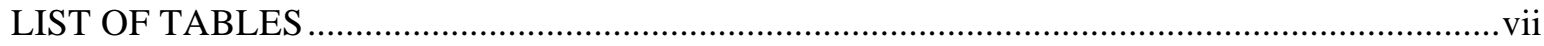

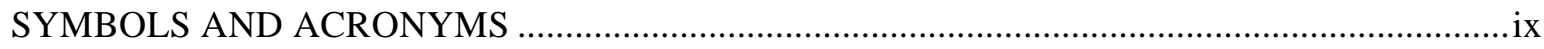

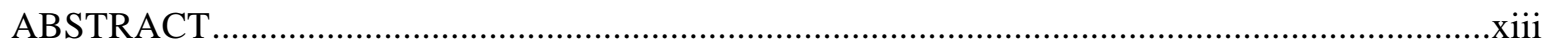

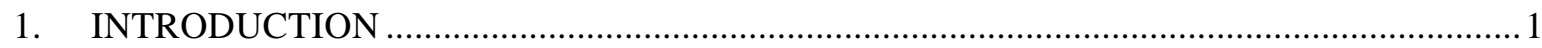

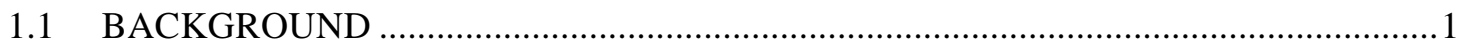

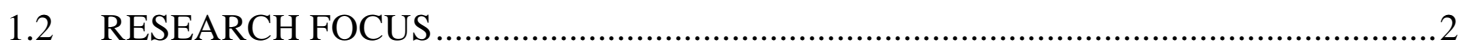

2. POWER GRID EXPANSION PLANNING CONSIDERING STOCHASTIC FACTORS

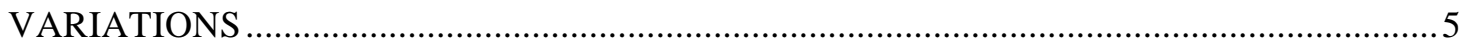

2.1 DATASET AND THE GENERATION-TRANSMISSION EXPANSION MODEL ......5

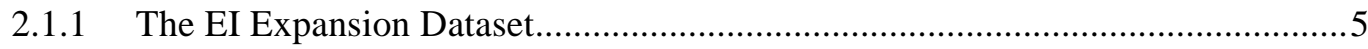

2.1.2 Generation-Transmission Expansion Co-Optimization Model .............................

2.1.3 Time Series Generation for Multi-Region Systems......................................... 10

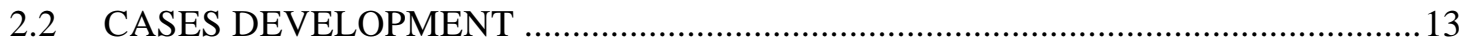

2.3 IMPACT OF WIND TEMPORAL DIVERSITY ON LT PLANNING .........................16

2.4 IMPACT OF WIND SPATIAL DIVERSITY ON LT PLANNING .............................20

2.5 IMPACT OF MORE DETAILED REPRESENTATION OF WIND POWER

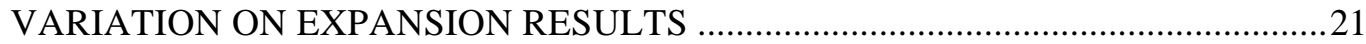

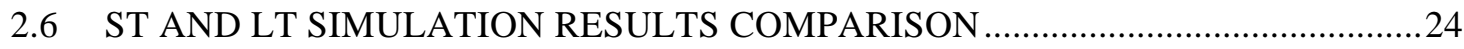

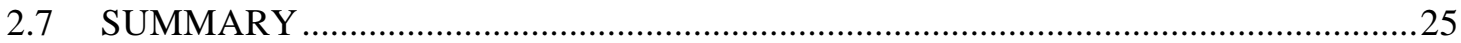

3. PRELIMINARY EXPERIMENTS ON HPC-BASED PARALLEL SIMULATIONS OF

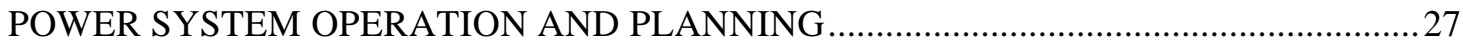

3.1 IMPACT OF MODEL COMPLEXITY ON COMPUTATION TIME IN LT

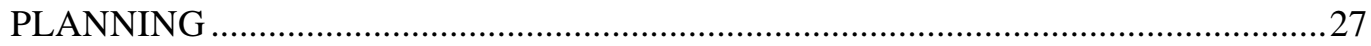

3.2 TESTING OF PRELIMINARY PARALLEL COMPUTATION TECHNIQUES IN

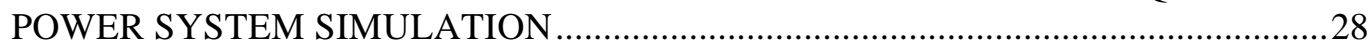

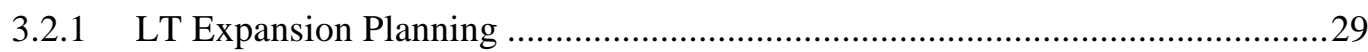

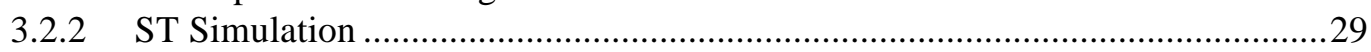

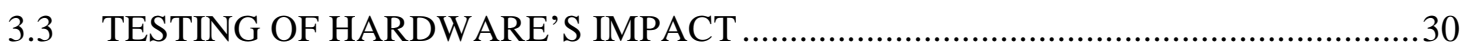

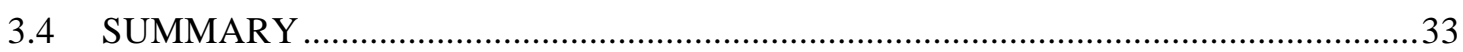

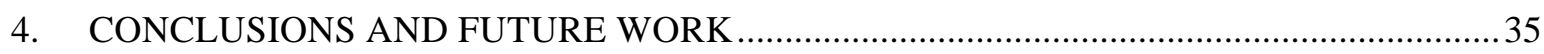

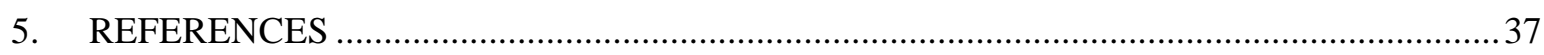





\section{LIST OF FIGURES}

Fig. 1. Regions of the US EI system (EI includes all non-gold regions) …...................................

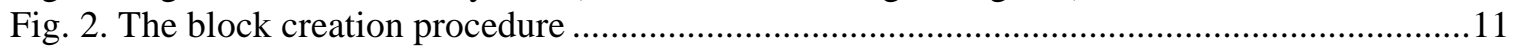

Fig. 3. SPP N wind variation representation in Case 20-Blk-Avg and Case 40-Blk-HiLo............14

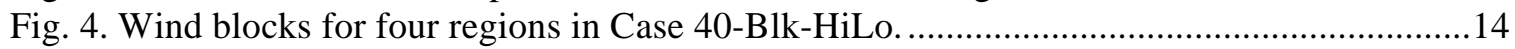

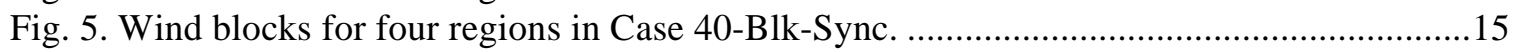

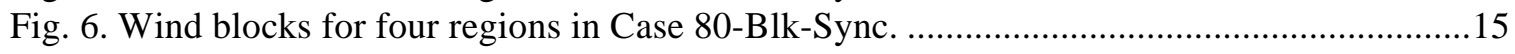

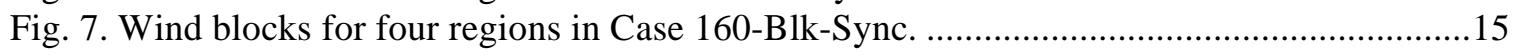

Fig. 8. Transmission capacity expansion of Case 20-Blk-Avg and Case 40-Blk-HiLo. ................17

Fig. 9. Annual generation expansion capacity of Case 20-Blk-Avg and Case 40-Blk-HiLo.........17

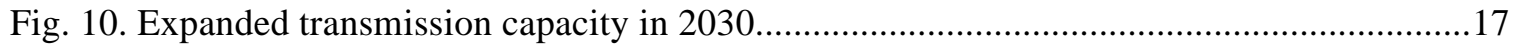

Fig. 11. The maximum and minimum flow for Case 20-Blk-Avg. ............................................18

Fig. 12. Maximum and minimum flow for Case 40-Blk-HiLo...................................................18

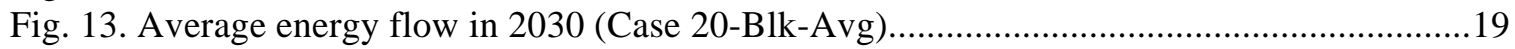

Fig. 14. Average energy flow in 2030 (Case 40-Blk-HiLo)......................................................19

Fig. 15. Expanded generation capacity by regions in 2030 (Case 20-Blk-Avg and Case 40-Blk-

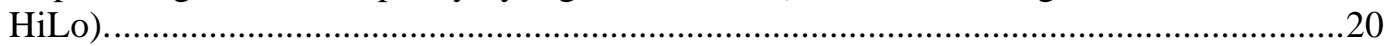

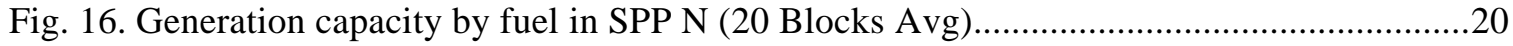

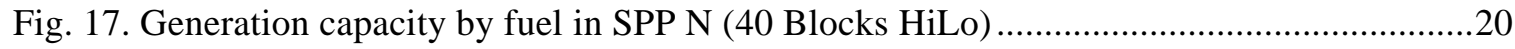

Fig. 18. Average energy flow in the year 2030 (Case 40-Blk-Sync) ...........................................21

Fig. 19. Transmission expansion in 20 Blocks, Case 40-Blk-HiLo, Case 40-Blk-Sync, and 80

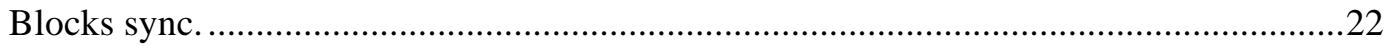

Fig. 20. The annual energy flow in 2030 (Case 20-Blk-Avg) (Linewidth is proportional to

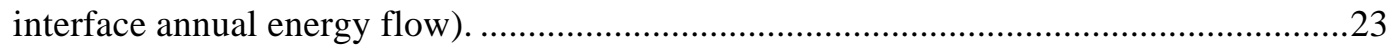

Fig. 21. The annual energy flow in 2030 (Case 160-Blk-Sync) (Linewidth is proportional to

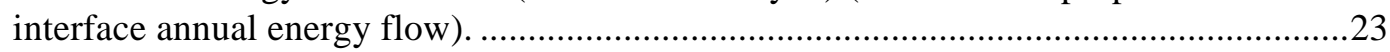

Fig. 22. Annual energy flow of the not-co-optimized case (Linewidth is proportional to interface

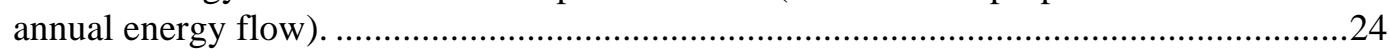

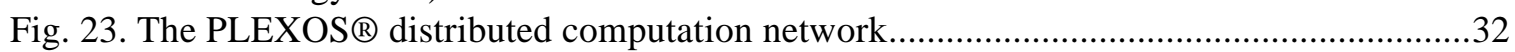





\section{LIST OF TABLES}

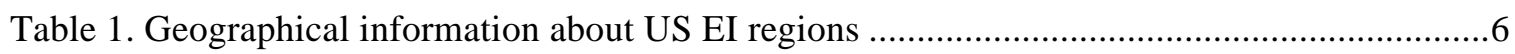

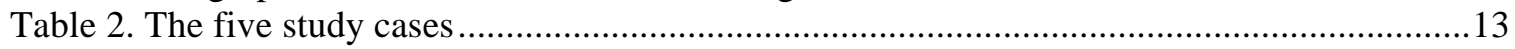

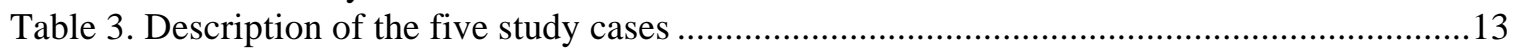

Table 4. Summary of the expansion results of Case 20-Blk-Avg and Case 40-Blk-HiLo .............16

Table 5. Cost breakdown of Case 20-Blk-Avg and Case 40-Blk-HiLo ........................................19

Table 6. Summary of the expansion results of Case 40-Blk-Sync ..............................................21

Table 7. Summary of expansion results of Case 80-Blk-Sync and Case 160-Blk-Sync ................22

Table 8. Expansion of gas and wind generation in PJM ROR and SPP N for the five cases .........23

Table 9. ST and LT simulation results in 2030 for the five cases .............................................24

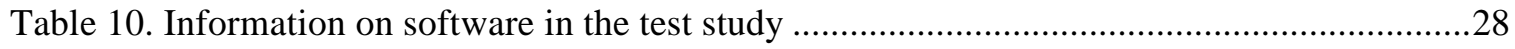

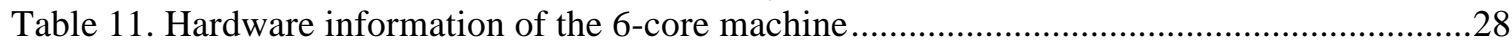

Table 12. Computation complexity when modelling various number of load levels in a three-node

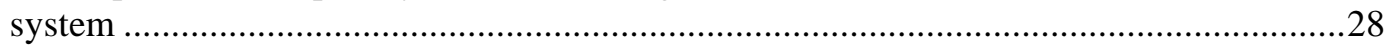

Table 13. Information on test cases for splitting samples in LT expansion ................................29

Table 14. Computation time of LT cases (splitting samples) on the 6-cores 12-thread machine...29

Table 15. Information on test cases for splitting chronology in ST operation simulation..............30

Table 16. Computation time of ST cases (splitting chronology) cases on the 6-core 12-thread

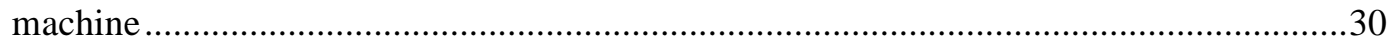

Table 17. Hardware information of the 20-core 40-thread machine ..........................................30

Table 18. Computation time of LT cases (splitting samples) on the 20-core 40-thread machine..31

Table 19. Computation time of each process in LT expansion optimization on machines with

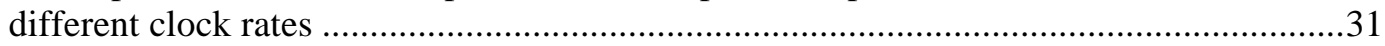

Table 20. Computation time of ST cases (splitting chronology) on different hardware ................32

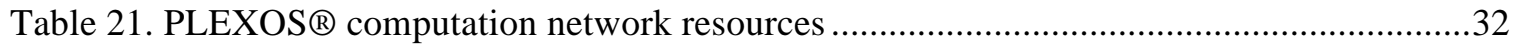





\section{SYMBOLS AND ACRONYMS}

\begin{tabular}{|c|c|}
\hline ACRONYMS & \\
\hline CRA & Charles River Associates \\
\hline EI & Eastern Interconnection \\
\hline EIPC & Eastern Interconnection Planning Committee \\
\hline EISPC & EI State's Planning Council \\
\hline ENT & Entergy (Entergy, central Missouri, east TX), (a region in EI) \\
\hline FRCC & Florida Reliability Coordinating Council - Florida minus panhandle, (a region in EI) \\
\hline GEP & Generation Expansion Planning \\
\hline GTEP & Generation-Transmission Co-expansion Planning \\
\hline HPC & High Performance Computing \\
\hline IESO & Independent Electricity System Operator, Ontario Canada, (a region in EI) \\
\hline LDC & Load Duration Curve \\
\hline LT & Long term \\
\hline MAPP CA & $\begin{array}{l}\text { Mid-continent Area Power Pool - Canada (Manitoba-Saskatchewan), (a region in } \\
\text { EI) }\end{array}$ \\
\hline MAPP US & $\begin{array}{l}\text { Mid-continent Area Power Pool - US (non-MISO regions in MT, ND, SD, MN, IA), } \\
\text { (a region in EI) }\end{array}$ \\
\hline MIP & Mixed-Integer Programming \\
\hline MISO IN & Mid-continent Independent System Operator - Indiana, (a region in EI) \\
\hline MISO MI & Mid-continent Independent System Operator - Michigan, (a region in EI) \\
\hline MISO MO-IL & $\begin{array}{l}\text { Mid-continent Independent System Operator - Missouri-Illinois (eastern MO, much } \\
\text { of IL), (a region in EI) }\end{array}$ \\
\hline MISO W & $\begin{array}{l}\text { Mid-continent Independent System Operator -West (parts of MT, ND, SD, MN, IA, } \\
\text { MN, WI), (a region in EI) }\end{array}$ \\
\hline MISO WUMS & Mid-continent Independent System Operator -Wisconsin-Upper Michigan \\
\hline $\mathrm{NE}$ & Nebraska, (a region in EI) \\
\hline NEISO & New England Independent System Operator, (a region in EI) \\
\hline MRN-NEEM & Multi-Region National-North American Electricity and Environment Model \\
\hline $\begin{array}{l}\text { Non-RTO } \\
\text { Midwest }\end{array}$ & $\begin{array}{l}\text { Non-Regional Transmission Operator Midwest (most KY, some OH public utilities), } \\
\text { (a region in EI) }\end{array}$ \\
\hline NPV & Net Present Value \\
\hline NYISO A-F & New York Independent System Operator - Upstate, (a region in EI) \\
\hline NYISO G-I & New York Independent System Operator - lower Hudson Valley, (a region in EI) \\
\hline NYISO J-K & $\begin{array}{l}\text { New York Independent System Operator - New York City-Long Island, (a region in } \\
\text { EI) }\end{array}$ \\
\hline PJM & $\begin{array}{l}\text { Independent System Operator for territory from Chicago to Virginia, formerly the } \\
\text { Pennsylvania-New Jersey-Maryland power pool, (a region in EI) }\end{array}$ \\
\hline PJM E & PJM Eastern Mid-Atlantic Area Council (NJ, DE, east MD), (a region in EI) \\
\hline PJM ROM & PJM Rest of Mid-Atlantic Area Council (east PA, DC, east MD), (a region in EI) \\
\hline PJM ROR & $\begin{array}{l}\text { PJM Rest of Regional Transmission Operator (north IL, OH, west PA, west MD, } \\
\text { WV, VA, east NC), (a region in EI) }\end{array}$ \\
\hline SOCO & Southern Company (GA, AL, east MS, west FL), (a region in EI) \\
\hline SPP N & Southwest Power Pool - North (Kansas, western Missouri), (a region in EI) \\
\hline SPP S & Southwest Power Pool South (Oklahoma, north TX, east NM, west AR, west LA) \\
\hline ST & Short-term \\
\hline TEP & Transmission Expansion Planning \\
\hline TVA & Tennessee Valley Authority (TN, north MS, north AL, south KY), (a region in EI) \\
\hline VACAR & Virginia-Carolina Sub-region - South Carolina, west North Carolina, (a region in EI) \\
\hline
\end{tabular}




\section{SYMBOLS-PARAMETERS}

\begin{tabular}{|c|c|}
\hline$C F_{y, r, s}$ & Wind capacity factor of generator $g$ in region $r$, year $y$, block $s$ \\
\hline$C_{e m m, r, y, g}$ & Emission cost for generator $g$, region $r$, year $y$ \\
\hline$C_{F O M, r, g}$ & Fixed operation and maintenance cost of generator $g$ located in region $r$ \\
\hline$C_{f u e l, y, r, g}$ & Fuel Price for generator $g$, region $r$, year $y$ \\
\hline$C_{G b u i l t, y, r, g}$ & Build cost of generator $g$ located in region $r$, year $y$ \\
\hline$C_{V O L L, r}$ & Value of lost load (energy shortage price) in region $r$ \\
\hline$C_{V O M, r, g}$ & Varying operation and maintenance cost of generator $g$ in region $r$ \\
\hline$C_{\text {Wheeling,l}}$ & The wheeling cost coefficient in interface $l$ \\
\hline$C_{X b u i l t, y, l}$ & Build cost of transmission interface $l$ in year $y$ \\
\hline$D F_{y}$ & Discount factor in the year $y$ \\
\hline$e_{r, g}$ & Emission coefficient of generator $g$ located in region $r$ \\
\hline$F_{F O R, r, g}$ & Forced outage rate of generator $g$ located in region $r$ \\
\hline$L^{\prime}$ & Load duration curves of raw chronological load data \\
\hline$L_{y, r, s}$ & Load in region $r$, block $s$, year $y$ \\
\hline$L_{y, s}$ & System-level load in block $s$, year $y$ \\
\hline$M F_{r, s}$ & Maintenance factor in region $r$ block $s$ \\
\hline$N_{L}$ & Number of transmission interfaces between regions \\
\hline$N_{Q}$ & Number of raw chronological curves \\
\hline$N_{R}$ & Number of regions \\
\hline$N_{r, G}$ & Number of generators (including both existing and candidates) in region $r$ \\
\hline$N_{Y}$ & Number of years in the planning horizon \\
\hline$N_{y, S}$ & Number of blocks in year $y$ \\
\hline$P_{\max , r, g}$ & Maximum generation capacity of generator $g$ in region $r$ \\
\hline $\bar{P}_{\max , r, g, s}$ & $\begin{array}{l}\text { Maximum generation of generator } g \text { after considering the forced and maintenance } \\
\text { outages discount in block } s \text { in region } r\end{array}$ \\
\hline$P_{l, \max }$ & Maximum transmission capacity of transmission interface $l$ \\
\hline$p r_{y, s}$ & Probability of blocks $s$ in year $y$ \\
\hline$p r_{q}^{\prime}$ & Probability of raw load curves $q$ \\
\hline$R_{H, r, g}$ & Heat rate of generator $g$ in region $r$ \\
\hline$R s_{y, r}$ & Required reserve margin in region $r$ year $y$ \\
\hline$R P S_{y, r}$ & Renewable portfolio standard percentage in region $r$ year $y$ \\
\hline$T$ & Number of hours in each year \\
\hline$x_{r, g}^{0}$ & Number of existing units of generator $g$ \\
\hline $\bar{x}_{\text {Gbuilt }, y, r, g}$ & Max unit number of annual expansion of generator $g$ in region $r$ \\
\hline
\end{tabular}


$X_{\text {MaxGbuilt }, r, g} \quad$ Maximum number of unit expansion of generation $g$

$X_{\text {MaxXbuilt }, l} \quad$ Maximum number of unit expansion of interface $l$

$\bar{x}_{X b u i l t, y, l} \quad$ Max unit number of annual expansion of interface $l$

$x_{r, l}^{0} \quad$ Number of existing lines of interface $l$

$\Omega_{r} \quad$ Set of the transmission interfaces of region $r$

SYMBOLS-VARIABLES

$I_{y, l, s} \quad$ Power flow of transmission interface $l$ in block $s$, year $y$

$P_{y, r, g, s} \quad$ Dispatch level of generating unit $g$ in blocks s, region $r$, and year $y$.

$P_{U S E, y, r, s} \quad$ Unserved power in region $r$, block $s$, year $y$

$x_{\text {Gbuilt }, y, r, g} \quad$ Expansion decision variable of generator $g$

$x_{X b u i l t, y, l} \quad$ Expansion decision variable of transmission interface $l$ in year $y$

\section{SYMBOLS-INDICES}

$g \quad$ Generator index

$l \quad$ Transmission interface index

$q \quad$ Raw chronological curve index

$r \quad$ Region index

$s \quad$ Block index

y $\quad$ Year index 



\begin{abstract}
Renewables are taking a large proportion of generation capacity in US power grids. As their randomness has increasing influence on power system operation, it is necessary to consider their impact on system expansion planning. To this end, this project studies the generation and transmission expansion cooptimization problem of the US Eastern Interconnection (EI) power grid with a high wind power penetration rate.
\end{abstract}

In this project, the generation and transmission expansion problem for the EI system is modeled as a mixed-integer programming (MIP) problem. This study analyzed a time series creation method to capture the diversity of load and wind power across balancing regions in the EI system. The obtained time series can be easily introduced into the MIP co-optimization problem and then solved robustly through available MIP solvers. Simulation results show that the proposed time series generation method and the expansion co-optimization model and can improve the expansion result significantly after considering the diversity of wind and load across EI regions. The improved expansion plan that combines generation and transmission will aid system planners and policy makers to maximize the social welfare.

This study shows that modelling load and wind variations and diversities across balancing regions will produce a significantly different expansion result compared with former studies. For example, if wind is modeled in more detail (by increasing the number of wind output levels) so that more wind blocks are considered in expansion planning, transmission expansion will be larger and the expansion timing will be earlier. Regarding generation expansion, more wind scenarios will slightly reduce wind generation expansion in the EI system and increase the expansion of other generation such as gas. Also, adopting detailed wind scenarios will reveal that it may be uneconomic to expand transmission networks for transmitting a large amount of wind power over a long distance in the EI system.

Incorporating more details of renewables in expansion planning will inevitably increase the computational burden. Therefore, high performance computing (HPC) techniques are urgently needed for power system operation and planning optimization. As a scoping study task, this project tested some preliminary parallel computation techniques such as breaking down the simulation task into several sub-tasks based on chronology splitting or sample splitting and then assigning these sub-tasks to different cores. Testing results show significant time reduction when a simulation task is split into several sub-tasks for parallel execution. 



\section{INTRODUCTION}

\subsection{BACKGROUND}

Power system expansion problems can be divided into three categories: generation expansion planning (GEP), transmission expansion planning (TEP), and generation-transmission co-expansion planning (GTEP). The objective of GEP is to decide the timing, size, technology (fuel), and location of generation build and retirement to minimize the cost while satisfying various constraints over the planning horizon. Similarly, TEP aims to determine the location, timing, and the sizes of future transmission lines. TEP can be considered together with GEP problems as GTEP problems [1]. GTEP problems can be modeled in two ways. One is the static method that views the entire planning horizon as a single stage and assumes that all investments happen at the beginning of the horizon. The other is the multi-stage (or dynamic) formulation that divides the planning horizon into sub-stages, providing more detailed results such as the timing information [2]. Further, GTEP can be studied under two environments: the market environment and the vertically integrated environment $[3,4]$.

Power grids are subjected to influences from stochastic factors, such as forced outages, load, renewables, and fuel cost uncertainties. With the increase of renewable penetration rates, the intermittency of wind and solar are becoming the major uncertainty sources in power systems. As studies predict that the United States could have around $27 \%$ of its electricity coming from renewables by 2030 [5], their fluctuations need to be considered in not only the operations stage but also the planning stage.

Some research focuses on single-stage expansion planning with uncertainties using conventional mathematical programming models $[6,7]$, but single-stage planning does not provide the timing of the expansion plan, missing operation information during the planning horizon. Some work considers mulitstage expansion planning with uncertainties, but expansion planning is limited to generation or transmission only [8-12]. In addition, the sizes of studied systems are usually small and the planning horizons are limited to several years due to problem complexity [13]. Most recent research adopts metaheuristic algorithms to solve the problem, which greatly improve the capability of solving nonlinear nonconvex optimization problems [14-18]. Nevertheless, heuristic algorithms may sometimes converge to local-optima $[19,20]$. To improve it, some research uses iterative methods to solve a series of mixedinteger linear programming problems [21].

Incorporating variations and uncertainties in expansion planning will produce a plan that has better economy and reliability performance during operations [22]. Recent progresses on robust optimization techniques can consider uncertain parameters in expansion optimization [23-25]. Two categories of robust optimization models have been successfully applied in power system expansion: probabilistic robust optimization models (e.g., stochastic optimization models) [24, 25] and non-probabilistic robust optimization models [23]. Probabilistic robust optimization models are capable of considering detailed parameter distribution such as wind and load variation. The most commonly used techniques for solving a stochastic optimization problem is formulating its deterministic equivalent through scenario construction. Besides considering all possible combinations of uncertainties [26, 27], some techniques to select representative scenarios have been applied, such as Taguchi's Orthogonal Array Testing [28]. As the other category of robust optimization, non-probabilistic robust optimization models are more adaptive to the representation of uncertainties. Therefore, they are applicable when parameter probabilistic distribution is not available. Such uncertainties include policy changes and market participant behaviors. Non-probabilistic optimization models usually adopt the Wald's maximum model to minimize the maximum adjustment cost and regret [23, 29]. The deficiencies of some robust optimization models include the computation complexity when applied to large-scale systems (for probabilistic robust optimization models) and the lack of ability to quantify the overall expected cost (for non-probabilistic 
robust optimization models). Besides, scenario construction to balance model accuracy and complexity is still an active research topic for both categories of methods [30].

Furthermore, in large power grids, the diversity of renewables and load across regions has significantly increased the necessity of energy exchange between regions to facilitate load and generation balancing on a larger scale. However, the variation of load and wind power in large multi-region systems has not been studied in existing GTEP models considering uncertainties [31].

Moreover, operation and planning simulations of modern power systems involve solving large-scale problems due to the system size or the long simulation timescale. Typically, these problems are complex programming/optimization problems involving many variables and constraints, which require a large amount of computation resources. Some research has studied parallel computing techniques to accelerate electromagnetic transient simulation [32], dynamic simulation [33], and Monte Carlo-based reliability assessment [34], but few studies have been done to investigate parallel computing techniques in system operation (market) simulation and long-term (LT) planning.

In this research, we use PLEXOS® to model the US EI generation and transmission planning problem as a mixed-integer programming (MIP) model, thus ensuring that the optimal plan can be obtained robustly and efficiently through leveraging existing commercial MIP solvers. In addition, a scenario creation method is proposed to capture the diversity of both load and wind power across regions for the EI system. Obtained scenarios that represent load and wind uncertainties and diversities can be easily introduced into the MIP problem and then solved to obtain the co-optimized generation and TEP. Applying these methods, the US EI system expansion co-optimization results are presented and discussed. As a scoping study on parallel computation techniques for system operation and optimization computation, some preliminary experiments conducted on several computation platforms at ORNL and the University of Tennessee (UTK) are documented.

\subsection{RESEARCH FOCUS}

This project focuses on the US EI system expansion planning considering wind power variation. In this study, EI multi-regional LT generation transmission expansion is modeled as a MIP problem, which can be solved by commercial solvers to obtain the optimal planning result. This MIP formulation can cooptimize generation and transmission expansion simultaneously to provide expansion timing information. This study also proposed a block (i.e., time-series data) creation method to incorporate the uncertainties and diversity of wind power and load in multiple regions. The obtained blocks can be easily integrated to the MIP problem for obtaining a better expansion plan compared with conventional methods. Applying the expansion model and the block generation method to EI during 2015 to 2030, this study aims to provide insight into the following issues:

1. How will the GEP and generation mix in different regions change if incorporating different levels of details on wind power variation?

2. How will the TEP change considering different levels of details on wind power variation?

3. What is the relationship between the timing of generation and transmission planning?

4. What are the possible methods and benefits in modeling the diversity of wind power resources in LT expansion planning?

In addition, this report documented some preliminary results on using parallel computation techniques for LT expansion optimizing and short-term (ST) market simulation. This study conducted a variety of test 
cases using various parallel computation techniques (split by chronology or samples), problem types (expansion planning or ST market simulation), and hardware platforms.

This report is organized as follows: Section 2 studies the impact of wind power modeling on generation and transmission expansion co-optimization; Section 3 presents preliminary results on testing parallel computation techniques; and Section 4 gives conclusions and future work. 



\section{POWER GRID EXPANSION PLANNING CONSIDERING STOCHASTIC FACTORS VARIATIONS}

This section focuses on studying the impact of wind power modeling on EI generation and transmission co-optimization planning for the years 2015-2030. This research adopted multiple methods to model wind uncertainties and made a comparative study based on the expansion results. Section 2.1 describes the EI expansion dataset and the generation-transmission co-optimization expansion model, as well as the proposed uncertainty modelling method for LT planning. Section 2.2 describes several cases developed in this study. Sections 2.3 to 2.5 present a comparative analysis on the developed cases based on their expansion results. Section 2.6 compares the LT and ST simulation results to verify the effectiveness of the proposed method. Section 2.7 is a summary.

\subsection{DATASET AND THE GENERATION-TRANSMISSION EXPANSION MODEL}

\subsubsection{The EI Expansion Dataset}

The US EI system consists of most of the power grid east of the Rocky Mountains, as shown in Fig. 1. The US EI multi-regional dataset is created by the Eastern Interconnection Planning Committee (EIPC) and Charles River Associates (CRA) [35] and translated to the PLEXOS format by Energy Exemplar [36]. This dataset partitions the EI system into 25 regions and the interfaces between adjacent regions [37]. The colored regions represent utilities, regional transmission operators, coordinating authorities, independent system operators, or other natural groupings based on the structure of the grid [37]. Between these regions, there are interfaces for power exchange. Table 1 shows the details of the EI regions.

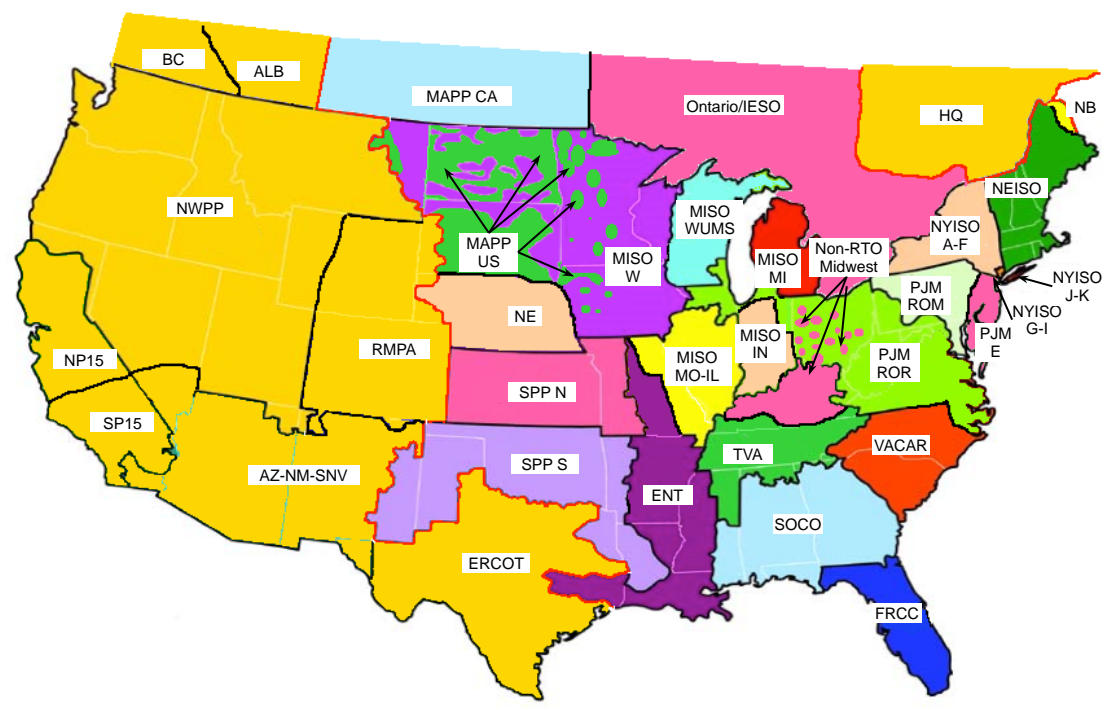

Fig. 1. Regions of the US EI system (EI includes all non-gold regions). 
Table 1. Geographical information about US EI regions

\begin{tabular}{lll}
\hline \multicolumn{1}{c}{ Region } & \multicolumn{1}{c}{ Description } & Territory \\
\hline MAPP CA & MAPP Canada (Manitoba-Saskatchewan) & Northwest \\
MAPP US & MAPP US (non-MISO regions in MT, ND, SD, MN, IA) & Northwest \\
MISO W & MISO West (parts of MT, ND, SD, MN, IA, MN, WI) & Northwest \\
MISO MO-IL & MISO Missouri-Illinois (eastern MO, much of IL) & Northwest \\
MISO WUMS & MISO Wisconsin-Upper Michigan & Northwest \\
MISO IN & MISO Indiana & Northwest \\
MISO MI & MISO Michigan & Central \\
\hline Non-RTO Midwest & Non-RTO Midwest (most KY, some OH public utilities) & Central \\
PJM ROR & PJM Rest of RTO (north IL, OH, west PA, west MD, WV, VA, east NC) & Central \\
PJM ROM & PJM Rest of MAAC (east PA, DC, east MD) & Central \\
PJM E & PJM Eastern MAAC (NJ, DE, east MD) & Northeast \\
\hline IESO & Ontario & Northeast \\
NYISO A-F & New York Upstate & Northeast \\
NYISO G-I & New York lower Hudson Valley & Northeast \\
NYISO J-K & New York City-Long Island & Northeast \\
NEISO & New England ISO & Southwest \\
\hline NE & Nebraska & Southwest \\
SPP N & SPP North (Kansas, western Missouri) & Southwest \\
SPP S & SPP South (Oklahoma, north TX, east NM, west AR, west LA) & Southwest \\
ENT & Entergy (Entergy, central Missouri, east TX) & Southeast \\
\hline TVA & Tennessee Valley Authority (TN, north MS, north AL, south KY) & Southeast \\
SOCO & Southern Company (GA, AL, east MS, west FL) & Southeast \\
VACAR & South Carolina, west North Carolina & Southeast \\
FRCC & Florida minus Panhandle &
\end{tabular}

Since 2010, EIPC had conducted a 4 year study on LT resources and transmission planning for the EI system. This study had created many future scenarios considering the uncertainties of generation, load, and transmission [38, 39]. EI State's Planning Council (EISPC) and DOE sponsored research on cooptimization of generation, transmission, and other resources [40]. The EI model and dataset used in this report is called the EIPC Phase 1 database, which is a bubble/pipe model representing 25 regions in EI and the interfaces between them. This database was developed by CRA and named the Multi-Region National-North American Electricity and Environment Model (MRN-NEEM) [41]. This database is simplified from the full nodal model from the EI system, focusing on generation expansion across EI regions and the transmission expansion on interfaces, while satisfying operation constraints and minimizing costs. More information on the model can be found in the MRN-NEEM document [42]. Some major characteristics of the model are summarized as below [37]:

1. Each region is treated as a node. All the generators and loads in each region are connected to one node, so no transmission lines within the region are modeled.

2. Each interface represents multiple transmission lines that connect two regions at multiple nodes, so interfaces are modeled as pipes with maximum transmission capacities. 
3. The model includes reliability constraints and environmental constraints, such as the minimum reserve margin and renewable portfolio standards.

4. The hourly demand, renewable generation, and fuel prices are aggregated into 20 "blocks" of different duration. Ten of the blocks represent the summer hours, while five blocks each denote the hours in winter and shoulder seasons.

The generation and transmission expansion model will be described in Section 2.1.2. This model is derived from the general model for expansion co-optimization in PLEXOS [36], aiming to maximize the social welfare or minimize the total cost. As the original EIPC dataset uses 20 block data for the demand, on-shore wind, off-shore wind, and solar in each year [35], detailed information on their variations across regions is not fully captured in optimization. In order to consider the realistic variation of demand, wind, and solar, this study modified the data by creating regional hourly demands, on-shore and off-shore wind generation, and solar production based on 2006 data used in EIPC study. Since hourly simulation for every year through the planning horizon is not possible in LT planning models, we have proposed a compromise method to consider the variations of wind power, which will be presented in Section 2.1.3.

\subsubsection{Generation-Transmission Expansion Co-Optimization Model}

The objective function of the expansion co-optimization model consists of three parts over the planning horizon: the expansion cost, the operation cost, and the emission cost. The expansion cost includes both the generation and the transmission expansion cost. The operation cost consists of the fixed operation cost and maintenance cost, the varying operation and maintenance cost, the fuel cost of generators, the value of the lost load, and the wheeling cost of transmission lines. Based on the generic MIP model in PLEXOS [36], the objective function of the GTEP problem can be expressed as follows:

$$
\begin{aligned}
& f=\sum_{y=1}^{N_{Y}} \sum_{r=1}^{N_{R}} \sum_{g=1}^{N_{r, G}} D F_{y} \cdot\left(C_{G b u i l t, y, r, g} \cdot x_{G b u i l t, y, r, g}\right) \\
& +\sum_{y=1}^{N_{Y}} \sum_{r=1}^{N_{R}} \sum_{g=1}^{N_{r, G}} D F_{y} \cdot\left[C_{F O M, r, g} \cdot P_{\mathrm{max}, r, g}\left(x_{r, g}^{0}+\sum_{y \leq y} x_{G b u i l t, y^{\prime}, r, g}\right)\right] \\
& +\sum_{y=1}^{N_{Y}} \sum_{r=1}^{N_{R}} D F_{y} \cdot T \sum_{s=1}^{N_{y, s}} p r_{y, s} \cdot \sum_{g=1}^{N_{r, G}} R_{H, r, g} \cdot C_{f u e l, y, r, g} \cdot P_{y, r, g, s}\left(x_{r, g}^{0}+\sum_{y^{\prime} \leq y} x_{G b u i l t, y^{\prime}, r, g}\right) \\
& +\sum_{y=1}^{N_{Y}} \sum_{r=1}^{N_{R}} D F_{y} \cdot T \sum_{s=1}^{N_{y, S}} p r_{y, s} \cdot \sum_{g=1}^{N_{r, G}} C_{V O M, r, g} \cdot P_{y, r, g, s}\left(x_{r, g}^{0}+\sum_{y^{\prime} \leq y} x_{G b u l t, y^{\prime}, r, g}\right) \\
& +\sum_{y=1}^{N_{Y}} \sum_{r=1}^{N_{R}} D F_{y} \cdot T \sum_{s=1}^{N_{y, S}} p r_{y, s} \cdot C_{V O L L, r} \cdot P_{U S E, y, r, s} \\
& +\sum_{y=1 l=1}^{N_{Y}} \sum_{L}^{N_{L}} D F_{y} \cdot\left(C_{X b u i l t, y, l} \cdot x_{X b u i l t, y, l}\right) \\
& +\sum_{y=1 l=1}^{N_{Y}} \sum_{L}^{N_{L}} D F_{y} \cdot T \sum_{s=1}^{N_{y, S}} p r_{y, s} \cdot C_{\text {Wheeling }, l} \cdot I_{y, l, s} \cdot\left(x_{r, l}^{0}+\sum_{y \leq y} x_{\text {Xbuilt }, y^{\prime}, l}\right) \\
& +\sum_{y=1}^{N_{Y}} \sum_{r=1}^{N_{R}} \sum_{g=1}^{N_{r, G}} D F_{y} \cdot\left[C_{e m m, y, r, g} \cdot e_{r, g} \cdot T \sum_{s=1}^{N_{y, s}} p r_{y, s} \cdot P_{y, r, g, s}\left(x_{r, g}^{0}+\sum_{y \leq y} x_{G b u i l t, y^{\prime}, r, g}\right)\right] \text {, }
\end{aligned}
$$

where $D F_{y}$ is the discount factor in year $y$. Before the end of the planning horizon, its value is 
$D F_{y}=\frac{1}{(1+d)^{y}} \quad y=1,2, \ldots, N_{Y}-1$

It is important that the expansion planning formulation does not inappropriately consider the end of the planning horizon to be the "end of time." Without considering the "end-year effects," the expansion plan would select to build generators with low build costs in the last several years, even if their marginal generation costs are high, so that the average cost in the horizon would be low. In order to consider the end-year effects, the last year of the horizon is repeated an infinite number of times [36]. Therefore, the discount factor in the end year considering the end-year effects is

$$
D F_{N_{Y}}=\frac{1}{(1+d)^{N_{Y}}}+\frac{1}{(1+d)^{N_{Y}+1}} \cdot \frac{1}{\left(1-\frac{1}{1+d}\right)},
$$

where

$\sum_{y=1}^{N_{Y}} \sum_{r=1}^{N_{R}} \sum_{g=1}^{N_{r, G}} D F_{y} \cdot\left(C_{G b u i l t, r, g} \cdot x_{G b u i l t, y, r, g}\right)$ is the generation built cost in all regions;

$\sum_{y=1}^{N_{Y}} \sum_{r=1}^{N_{R}} \sum_{g=1}^{N_{r, G}} D F_{y} \cdot\left[C_{F O M, r, g} \cdot P_{\max , r, g}\left(x_{r, g}^{0}+\sum_{y \leq y^{\prime}} x_{G b u i l t, y^{\prime}, r, g}\right)\right]$ is the fixed operation and maintenance cost in all regions (in $\$ / \mathrm{kW} /$ year, forming part of the unit annual fixed cost charge);

$\sum_{y=1}^{N_{Y}} \sum_{r=1}^{N_{R}} D F_{y} \cdot T \sum_{s=1}^{N_{y, s}} p r_{y, s} \cdot \sum_{g=1}^{N_{r, G}} R_{H, r, g} \cdot C_{f u e l, y, r, g} \cdot P_{y, r, g, s}\left(x_{r, g}^{0}+\sum_{y \leq y} x_{G b u i l t, y^{\prime}, r, g}\right)$ denotes the fuel cost.

$\sum_{y=1 r=1}^{N_{Y}} \sum_{r}^{N_{R}} D F_{y} \cdot T \sum_{s=1}^{N_{y, S}} p r_{y, s} \cdot \sum_{g=1}^{N_{r, G}} C_{V O M, r, g} \cdot P_{y, r, g, s}\left(x_{r, g}^{0}+\sum_{y \leq y} x_{G b u i l t, y^{\prime}, r, g}\right)$ denotes the varying operation and

maintenance cost (in $\$ / \mathrm{kW} /$ year, representing an incremental cost of generation used to recover regular equipment replacement and servicing costs that are a direct function of generation, e.g. wear and tear);

$\sum_{y=1 r=1}^{N_{Y}} \sum_{r=1}^{N_{R}} D F_{y} \cdot T \sum_{s=1}^{N_{y, S}} p r_{y, s} \cdot C_{V O L L, r} \cdot P_{U S E, y, r, s}$ denotes the value of lost load; $\sum_{y=1 l=1}^{N_{Y}} \sum_{L}^{N_{L}} D F_{y} \cdot\left(C_{X b u i l t, y, l} \cdot x_{X b u i l t, y, l}\right)$ is the transmission expansion cost of all interfaces;

$\sum_{y=1 l=1}^{N_{Y}} \sum_{L}^{N_{L}} D F_{y} \cdot T \sum_{s=1}^{N_{y, S}} p r_{y, s} \cdot C_{\text {Wheeling }, l} \cdot I_{y, l, s} \cdot\left(x_{r, l}^{0}+\sum_{y=y} x_{X b u i l t, y^{\prime}, l}\right)$ is the wheeling cost of transmission lines; and $\sum_{y=1}^{N_{Y}} \sum_{r=1}^{N_{R}} \sum_{g=1}^{N_{r, G}} D F_{y} \cdot\left[C_{e m m, y, r, g} \cdot e_{r, g} \cdot T \sum_{s=1}^{N_{y, s}} p r_{y, s} \cdot P_{y, r, g, s}\left(x_{r, g}^{0}+\sum_{y^{\prime} \leq y} x_{G b u i l t, y^{\prime}, r, g}\right)\right]$ is the emission cost.

The objective function (2.1) is the net present value of the sum of all of the system's cost items over the planning horizon considering the end-year effects. A practical expansion plan should also satisfy various planning and operation constraints. Constraints considered in this expansion planning problem include the following.

- Power balance constraint

In each region, the sum of generation output, unserved demand, and interface interchange should be equal to the demand for all regions within the planning horizon. 
$\sum_{g=1}^{N_{r, G}} P_{y, r, g, s}+P_{U S E, y, r, s}+\sum_{l \in \Omega_{r}} I_{y, l, s}=L_{y, r, s} \quad \forall y, \forall r$

where $\sum_{g=1}^{N_{r, G}} P_{y, r, g, s}$ denotes generation of all generators in region $r$ under the block $s ; \sum_{l \in \Omega_{r}} I_{y, l, s}$ denotes the power interchange through all the interfaces of region $r$.

- Maximum expansion constraint for generation

Due to resource limitation (such as maximum exploitable resources for hydro and nuclear power plants), the number of generator expansions in each region should be within its upper limit.

$$
\sum_{y \leq N_{Y}} x_{\text {Gbuilt }, y, r, g} \leq X_{\text {MaxGbuilt }, r, g} \quad \forall g, \forall r
$$

- Maximum expansion constraint for transmission

Due to the right-of-way limitation, the number of expanded interfaces should be within its upper limit.

$$
\sum_{y \leq N_{Y}} x_{X b u i l t, y, l} \leq X_{\text {MaxXbuilt }, l} \quad \forall l
$$

- Integer constraint

The number of built generators and interfaces should be integers.

$$
x_{\text {Gbuilt }, y, r, g} \in N ; x_{X b u i l t, y, l} \in N
$$

- Expansion speed constraint

Due to the construction resource limitation, the annual expansion speed of generators and transmission lines should be within their upper limits.

$$
x_{\text {Gbuilt }, y, r, g} \leq \bar{x}_{\text {Gbuilt }, y, r, g} ; \quad x_{X b u i l t, y, l} \leq \bar{x}_{X b u i l t, y, l} \quad \forall y, \forall g, \forall r, \forall l
$$

- Capacity discount considering forced and maintenance outages

In each region, the available capacity is usually less than the installed capacity due to forced and maintenance outages. The capacity discount is determined by the maintenance outage rate, the maintenance factor, and the forced outage rate. Since maintenance tasks are usually scheduled in off-peak periods, the maintenance factor $M F_{r, s}$ is related to the load level in block $s$.

$$
\bar{P}_{\max , r, g, s} \leq\left(1-F_{M O R, r, g} \cdot M F_{r, s}+F_{F O R, r, g}\right) \cdot P_{\max , r, g} \cdot\left(x_{r, g}^{0}+\sum_{y^{\prime} \leq y} x_{G b u i l t, y^{\prime}, r, g}\right) \quad \forall g, \forall r
$$

- Regional reserve capacity constraint 
The reserve capacity of each region should be larger than a predetermined level for regulation and contingency requirements. The reserve capacity level is set to be able to cover the energy imbalance caused by any single generator or interface failure associated with this region.

$$
\bar{P}_{\max , r, g, s}\left(x_{r, g}^{0}+\sum_{y^{\prime} \leq y} x_{G b u i l t, y^{\prime}, r, g}\right) \geq L_{y, r, s}-p \sum_{l \in \Omega_{r}} I_{y, l, s}+R s_{y, r} \quad \forall y, \forall r
$$

- Interface capacity constraint

To facilitate interregional analysis based on information provided by regional planning authorities [35], resources inside a region are considered to be connected to a notional node. Nodes that are not associated with a region are considered regions by themselves. The interface power flows between regions are modeled by the transportation model $[43,44]$. Interface flows should satisfy their limits.

$$
-P_{l, \max }\left(x_{r, l}^{0}+\sum_{y^{\prime} \leq y} x_{X b u i l t, y^{\prime}, l}\right) \leq I_{y, l, s} \leq P_{l, \max }\left(x_{r, l}^{0}+\sum_{y^{\prime} \leq y} x_{X b u i l t, y^{\prime}, l}\right) \quad \forall y, \forall l
$$

\section{- Regional renewable portfolio constraint}

In those regions with renewable portfolio constraints, the percentage of renewables in the total installed generation capacity should be higher than a predetermined value.

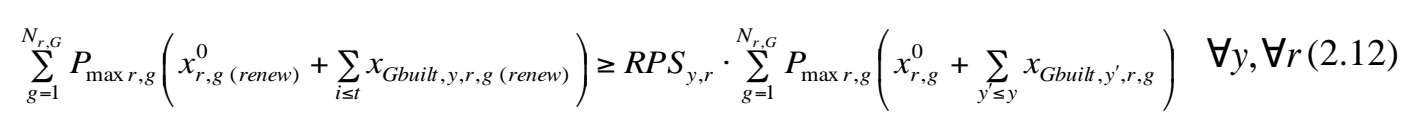

- Wind resource constraint

The output of wind turbine generators is restricted by the available wind resource.

$$
P_{y, r, g, s(\text { wind })} \leq C F_{y, r, s} \bar{P}_{\text {max }, r, g(\text { wind })} \quad \forall y, \forall r, \forall g
$$

The objective function (1) and constraints (4)-(13) comprise a MIP problem that minimizes the cost of the expansion plan while satisfying multiple operational and environmental constraints.

Except for the fixed operation and maintenance cost, all the operation-related cost items in the objective function are related to the load and wind levels. The obtained expansion plan is the optimal considering all the stochastic factors, such as load and wind. Section 2.1.3 will show the methodology of integrating these stochastic factors into the generation and transmission expansion co-optimization problem.

\subsubsection{Time Series Generation for Multi-Region Systems}

In LT planning, hourly load data are usually aggregated and represented by blocks for computation complexity consideration. Typical load aggregation methods include the load duration curve method and the load curve fitting method. The resulting load representation will have a limited number of blocks in each year that represent different levels of demand and corresponding time duration.

For multi-region expansion planning, load blocks should be synchronized (the load data in one block have the same chronological time stamps for all regions) in order to preserve the load diversity across regions. Load blocks synchronization is accomplished through the following steps: 
1) Obtain the load duration curves $\left\{L^{\prime}\right\}_{N Q}$ of the system based on the future demand data obtained by load forecasting. The total number of the curves is $N_{Q}$, and each curve has probability $p r_{q}^{\prime}$.

2) Simultaneously optimize the target load scenarios $\left\{L_{y, s}\right\}_{N y, s}$ and probabilities $p r_{y, s}$ using the least-squares fitting approach, i.e., minimizing $\sum_{s=1}^{N_{y, s}} p r_{y, s}\left(\sum_{q=1}^{N_{Q}} p r_{q}^{\prime}\left(L_{y, s}-L^{\prime}\right)^{2}\right)$. (In particular, the maximum demand period of all load duration curves are preserved to form a scenario.) Record the consequent chronology-to-scenario mapping function $\Theta_{L}$.

3) Use $\Theta_{L}$ to rearrange the load data of each region.

4) Calculate the expected value of load data for each scenario and region. The scenario probabilities are inherited from Step 2).

Similar to the load, wind power variations in each region should also be considered in expansion planning, especially if wind is a significant fraction of the total generation. Each time block can be further divided into several sub-blocks with different wind output levels. In addition, since large power systems usually have wide geographic areas, wind resources could vary greatly in different regions at the same time point. Therefore, a block creation method needs to be developed that minimizes the number of blocks while still capturing information about multi-regional chronological wind output. The expansion model can then include system-level high- and low-wind scenario blocks in each demand level, as well as the diversity of wind resources across regions. To meet this requirement, a generic block creation method [45] for multiple stochastic variables in expansion planning is developed and shown in Fig. 2. A description of the steps follows.

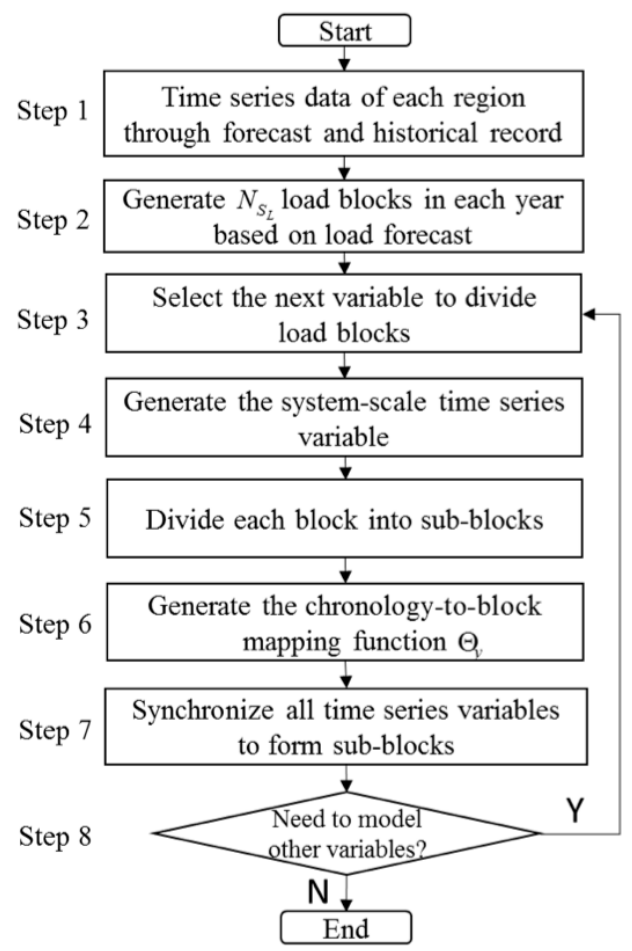

Fig. 2. The block creation procedure 
- Step 1. Prepare the time series load, wind, or solar data over the planning horizon based on forecast data or scaling historical data.

- Step 2. Use the least-squares fitting method to form $N_{S_{L}}$ load scenarios, namely, $s_{L 1}, s_{L 2}, \ldots, S_{L N_{S L}}$, in each year, that is, $N_{S_{L}} \times N_{Y}$ scenarios in total over the planning horizon. The probability of each scenario is denoted by $p r_{y, s}$.

- Step 3. Select the next stochastic factor $v_{k}$ ( $v_{k}$ can be wind, solar, or cost uncertainty depending on the study focus or the influences on expansion. In the following steps, wind is described for convenience).

- Step 4. Create the system-scale time-series data of the wind capacity factor. In many large-scale power grids, wind farms (both existing and candidate) are centralized in certain regions with rich wind resource. The time-series wind capacity factors in these regions are better indicators of the wind output of the whole system compared with those of regions that have much less wind power. Thus, the weighted time-series values of wind capacity factor is used to form a system-scale chronological attribute - the multi-region system wind capacity factor

$C F_{\text {system }, t}=\sum_{r=1}^{N_{R}} W_{i n d C a p}^{*} \cdot C F_{r, t}$,

where WindCap ${ }_{r}^{*}$ denotes the rough target wind generation capacity in region $r$, which can be obtained through estimation or iteratively updating from the previous planning result. $C F_{r, t}$ is the time series wind capacity factor in region $r . N_{R}$ is the number of regions.

- Step 5. Divide each load scenario $s_{L l}$ into $N_{S_{W}}$ sub-scenarios of different wind scenarios, namely, $s_{L, W 1}, s_{L, W 2}, \ldots, s_{L, W N_{S W}}$. Each sub-scenario represents a certain range of those wind capacity factors happening during the corresponding period of the load scenario. For instance, the lowest $1 / N_{S_{W}}$ percent system-scale chronological wind capacity factor data $\left(C F_{\text {system }, t}\right)$ in the load scenario $s_{L l}$ is aggregated in sub-scenario $s_{L l, W 1}$, and the highest $1 / N_{S_{W}}$ percent data is aggregated in $s_{L l, W N_{S W}}$. The probability of each sub-scenario is $p r_{y, s} / N_{S_{W}}$. (An alternative and more computation-intensive method to obtain the sub-scenarios and probabilities using the least-squares approach is described in "Load scenario synchronization for multi-region power grids".)

- Step 6. Generate the chronology-to-block mapping function $\Theta_{y}$. In forming each sub-block, the correspondence between the original chronological data $C F_{r, t}$ and each sub-block $s_{L l, W w}$ is recorded as the chronology-to-block mapping function denoted by $\Theta$. $\Theta$ differs from year to year, and it is denoted by $\Theta_{y}$ for year $y$.

- Step 7. According to the chronology-to-block mapping function $\Theta_{y}$, synchronize all the chronological data (load, wind, and solar) of all regions to form $\left(N_{S_{L}} \times N_{S_{W}}\right) \times N_{Y}=N_{S} \times N$ blocks.

- Step 8. Check if there are other stochastic factors (such as solar in high solar systems) that need to form sub-blocks in expansion planning. If so, return to Step 3. Otherwise, we end the block generation process. 
This block forming method can model multiple coupled stochastic variables for LT expansion planning through creating fewer blocks with various time durations. Moreover, the formed blocks can be directly put into the mixed-integer programming model and then solved through commercial solvers to obtain the optimal GTEP. It should be noted that Step 7 synchronizes all the stochastic factors, so those factors that have not been selected to further divide blocks still have different values in different blocks. The difference is that their blocks are passively formed (or synchronized) since their information is not specifically used to form sub-blocks due to the computation complexity that restricts the number of blocks in the optimization problem.

\subsection{CASES DEVELOPMENT}

As mentioned in the previous section, wind, solar, and load data in each year in the EIPC Phase 1 dataset are represented by 20 blocks ( 10 blocks for peak load periods, five for winter periods, and five for offpeak periods) [35]. In this research, we developed four additional cases to examine the impact of changing the block size and the aggregation technique on (1) transmission expansion and flows; (2) wind generation location and timing; and (3) costs. The developed cases are summarized in Table 2. Descriptions of those cases are provided in Table 3.

Table 2. The five study cases

\begin{tabular}{|l|l|l|}
\hline \multicolumn{1}{|c|}{ Case category } & \multicolumn{1}{|c|}{ Cases } & \multicolumn{1}{c|}{ Simulations } \\
\hline Base Case & Co-optimized 20 Blocks & LT 2015-2030; ST in 2030 \\
\hline \multirow{5}{*}{ Cases Developed } & $\begin{array}{l}\text { Co-optimized 40 Blocks HiLo } \\
\text { Co-optimized 40 Blocks Synchronized } \\
\end{array}$ & Co-optimized 80 Blocks Synchronized \\
& Co-optimized 160 Blocks Synchronized & \\
\hline
\end{tabular}

Table 3. Description of the five study cases

\begin{tabular}{|l|l|}
\hline \multicolumn{1}{|c|}{ Case name } & \multicolumn{1}{c|}{ Case description } \\
\hline $\begin{array}{l}\text { Co-optimized 20 Blocks } \\
\text { (Case 20-Blk-Avg) }\end{array}$ & $\begin{array}{l}\text { The base case has 20 load blocks in each year } \\
\text { Wind is the average value in each load block }\end{array}$ \\
\hline $\begin{array}{l}\text { 40 Blocks HiLo } \\
\text { (Case 40-Blk-HiLo) }\end{array}$ & $\begin{array}{l}\text { Split each block in two equal number of hours } \\
\text { Average of high wind in a half and average of low wind in the other half } \\
\text { (wind data are not synchronized). }\end{array}$ \\
\hline $\begin{array}{l}\text { 40 Blocks Synchronized } \\
\text { (Case 40-Blk-Sync) }\end{array}$ & $\begin{array}{l}\text { Determine hours of high- and low-wind capacity factors based on the } \\
\text { weighted average system-scale data in each of the 20 load blocks } \\
\text { Synchronize wind, solar, and load to the average of the region's values in } \\
\text { those hours }\end{array}$ \\
\hline $\begin{array}{l}\text { 80 Blocks Synchronized } \\
\text { (Case 80-Blk-Sync) }\end{array}$ & $\begin{array}{l}\text { Break each load block into four quartiles based on the weighted average } \\
\text { system-scale data in each of the 20 load blocks } \\
\text { Synchronize all regions' wind, solar, load, and fuel prices to those hours }\end{array}$ \\
\hline $\begin{array}{l}\text { 160 Blocks Synchronized } \\
\text { (Case 160-Blk-Sync) }\end{array}$ & $\begin{array}{l}\text { Break each load block into eight quartiles based on the weighted average } \\
\text { system-scale data in each of the 20 load blocks } \\
\text { Synchronize all regions' wind, solar, load, and fuel prices to those hours }\end{array}$ \\
\hline
\end{tabular}

Using the proposed method, each load block can be further divided into several sub-blocks with different wind output levels. Fig. 3 shows the wind capacity factor of the Southwest Power Pool - North (SPP N) region in three cases' datasets: (1) hourly; (2) Case 20-Blk-Avg; and (3) Case 40-Blk-HiLo. It shows that the output profile of Case 20-Blk-Avg is smoother compared with the raw chronological data. Case 40-Blk-HiLo 
preserves some wind power variation information as it splits each original block into two blocks with an equal number of hours that represent high and low wind in half.

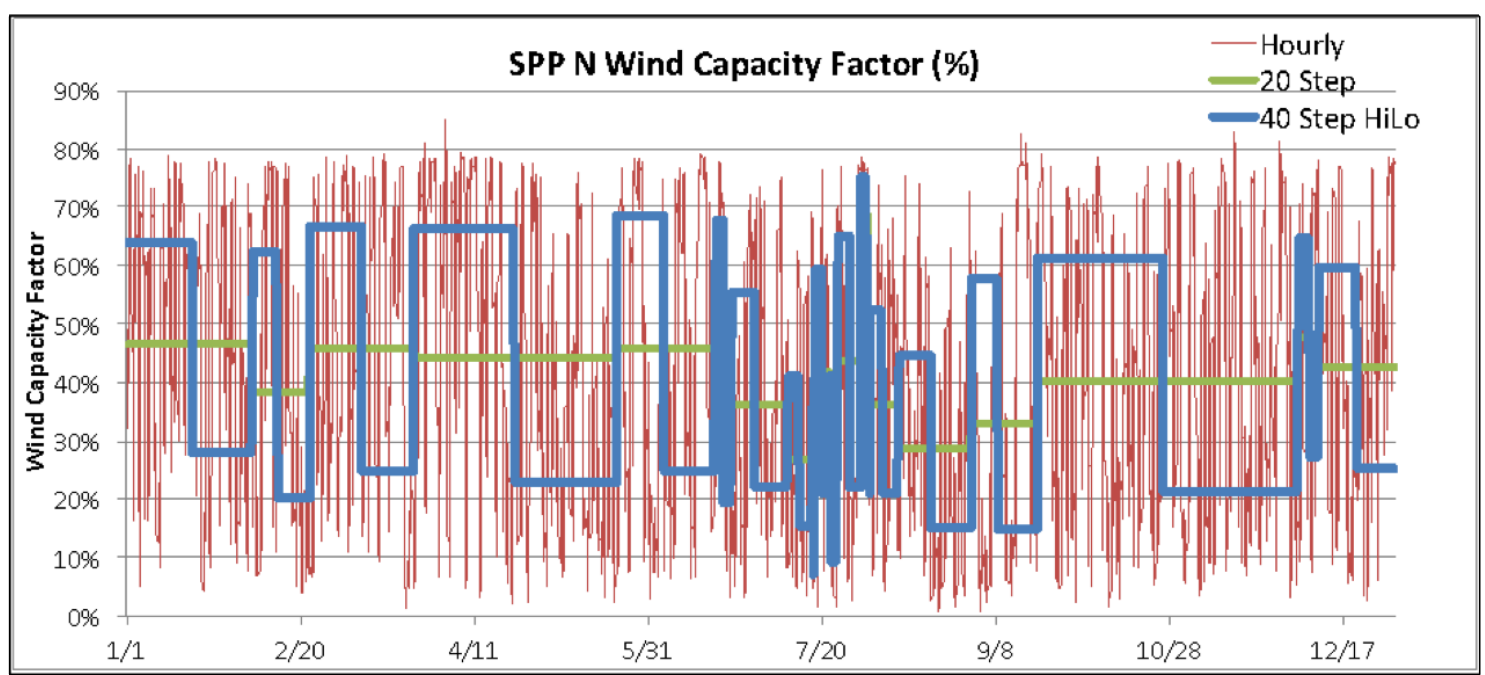

Fig. 3. SPP N wind variation representation in Case 20-Blk-Avg and Case 40-Blk-HiLo.

Fig. 4 shows the wind blocks of four regions in Case 40-Blk-HiLo. It is assumed in Case 40-Blk-HiLo that wind in all regions is highly correlated. In other words, the high wind is supposed to happen simultaneously in all regions, as does the low wind.

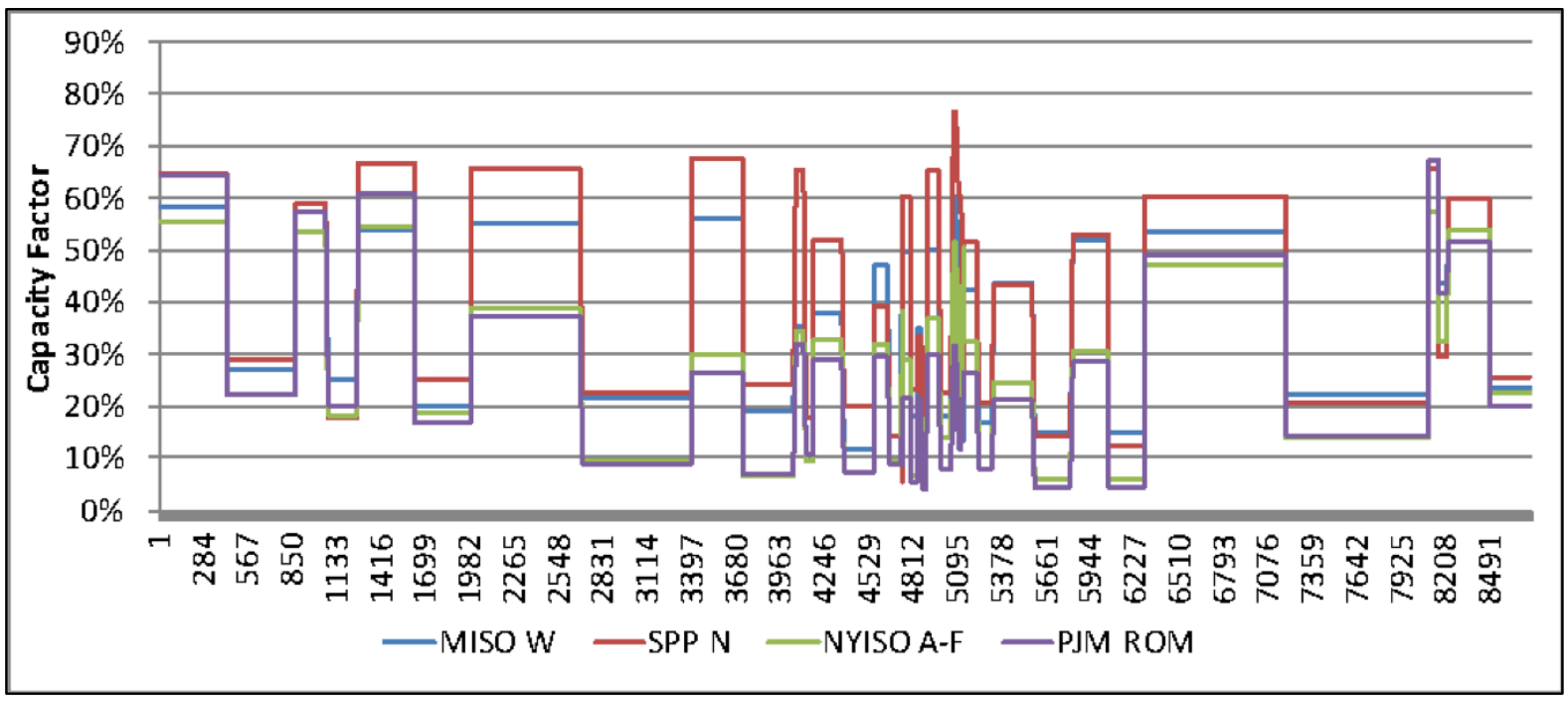

Fig. 4. Wind blocks for four regions in Case 40-Blk-HiLo.

Nevertheless, based on the hourly data, it is clear that due to weather and geographic factors, the half periods with high wind output in one region do not totally overlap with those in another region. It is also noted that nearby wind regions have more synchronicity on wind output levels, while ones further away have less. Therefore, we developed Case 40-Blk-Sync based on the method in Section 2.1.3 to capture the correlation degree of wind output between regions. Wind variations in four regions represented in Case 40-Blk-Sync are shown in Fig. 5. 


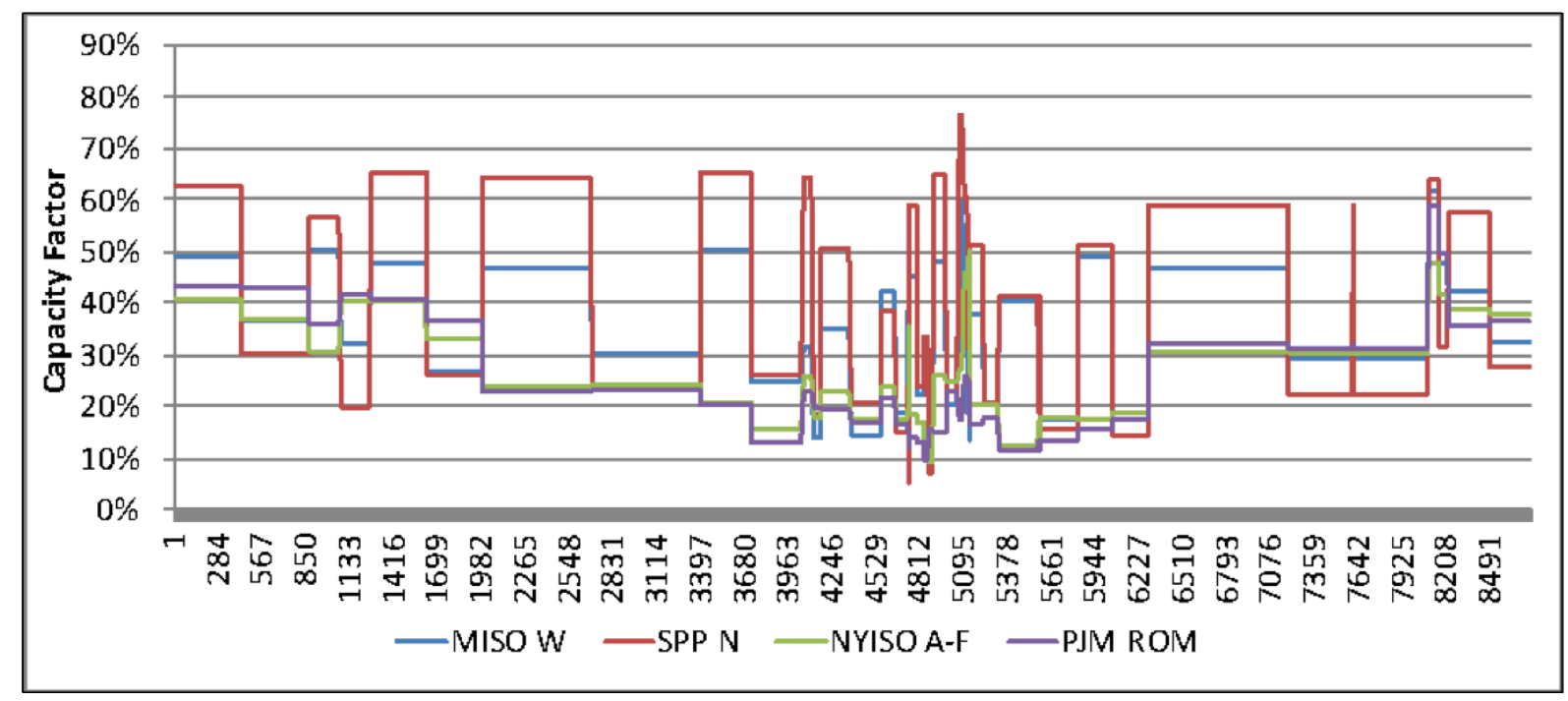

Fig. 5. Wind blocks for four regions in Case 40-Blk-Sync.

It should be noticed that the hourly solar, load, and fuel price data are also synchronized with the wind data to form their 40 synchronized blocks for LT expansion planning. In this way, the wind, solar, load, and fuel prices keep their synchronization in Case 40-Blk-Sync. Variables that have not been selected to further divide blocks still have different values in each block. The difference is that their blocks are passively formed (or synchronized) since their information is not specifically used to form sub-blocks due to the computation complexity that restricts the number of blocks in the optimization problem. Further, this method is used to extend it to Case 80-Blk-Sync and Case 160-Blk-Sync to capture more details in wind output. As an example, wind output blocks in the four regions are shown in Fig. 6 and Fig. 7.

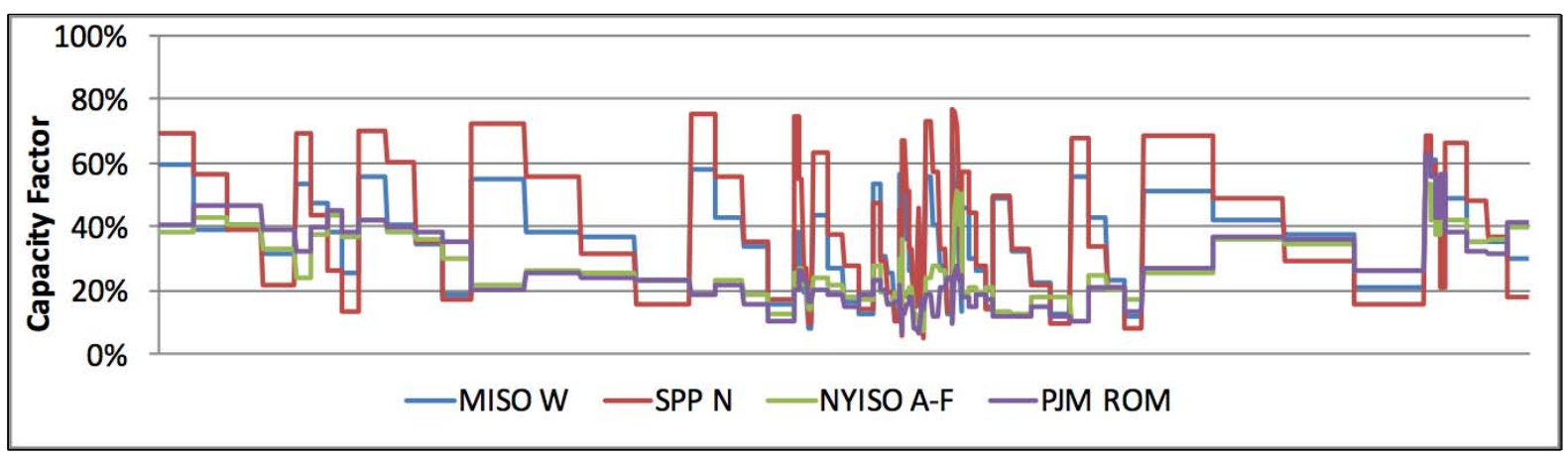

Fig. 6. Wind blocks for four regions in Case 80-Blk-Sync.

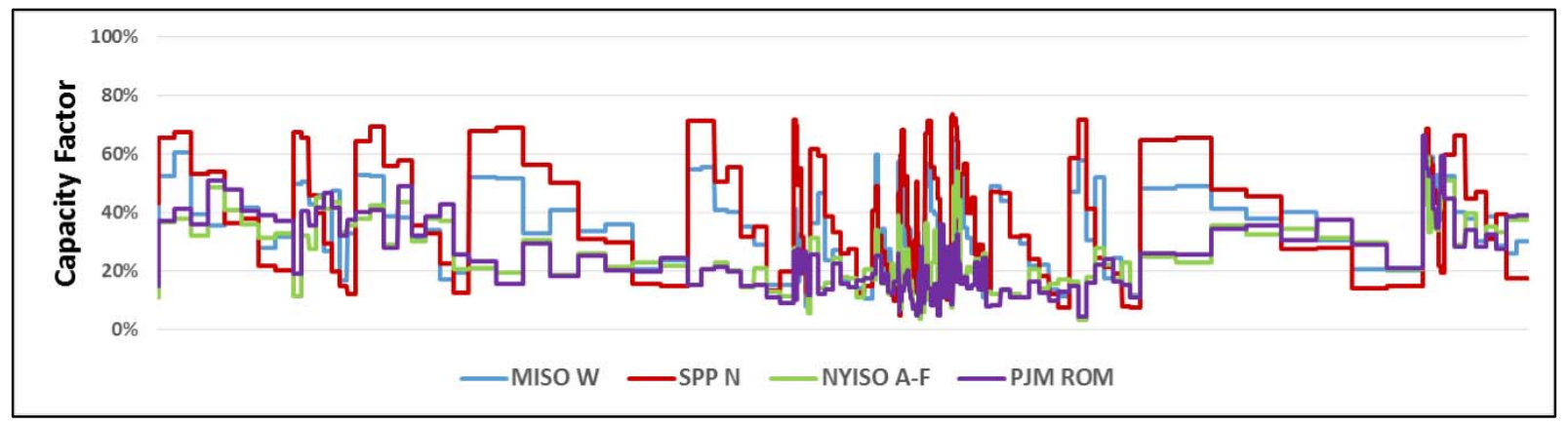

Fig. 7. Wind blocks for four regions in Case 160-Blk-Sync. 


\subsection{IMPACT OF WIND TEMPORAL DIVERSITY ON LT PLANNING}

The EI multi-regional dataset and the generation and transmission expansion problem are modeled in PLEXOS [36], and the problem is solved by the MIP solver - Xpress-MIP 26.01.04. The optimization is performed on a server with two Intel Xeon E5-2470.0 V2 CPUs (2.40 GHz, 40 cores) and $128 \mathrm{~GB}$ memory.

Case 20-Blk-Avg uses the average wind speed during each step, neglecting many scenarios in which the actual wind speed could be higher or lower. However, these scenarios may have significant impacts on the transmission and GEP for future power grids, especially when the system has a high wind penetration rate. Table 4 is a summary of the expansion results of Case 20-Blk-Avg and 40-Blk-Sync.

Table 4. Summary of the expansion results of Case 20-Blk-Avg and Case 40-Blk-HiLo

\begin{tabular}{lccc}
\hline \multicolumn{1}{c}{ Expansion results } & Case 20-Blk-Avg & 40-Blk-HiLo & \% Change \\
\hline Wind Capacity Built (GW) & 262 & 218 & $-16.5 \%$ \\
Total Wind Capacity (GW) & 304 & 260 & $-14 \%$ \\
Wind Generation in 2030 (GWh) & 917 & 768 & $-16 \%$ \\
Transmission expansion (GW) & 82.4 & 86.1 & $+4.5 \%$ \\
Transmission Build Cost (NPV) & $\$ 20.2$ billion & $\$ 26.1$ billion & $+29 \%$ \\
Gen Build Cost (NPV) & $\$ 649$ billion & $\$ 595$ billion & $-8 \%$ \\
Emissions in 2030 (million tons) & 305 & 365 & $+4 \%$ \\
Fuel Offtake in 2030 2030 (million GBTU) & 17.1 & 18.2 & $+2 \%$ \\
Wind Penetration in 2030 (energy) & $30.1 \%$ & $24.8 \%$ & $-16 \%$ \\
Wind Penetration in 2030 (capacity) & $31.4 \%$ & $28.3 \%$ & $-10 \%$ \\
\hline
\end{tabular}

From Table 4, it can be seen that in Case 40-Blk-HiLo, wind generation expansion is smaller while transmission expansion is larger compared with Case 20-Blk-Avg. The reason is that the capacity value of wind power decreases when more detailed wind power is modeled (divided into high and low output blocks in Case 40-Blk-HiLo), and the system tends to rely more on gas generation when more detailed variation of wind power is considered. In addition, high-wind scenarios need more interregional transmission capacity, thus requiring higher transmission expansion.

The annual transmission expansion capacities of the two cases are shown in Fig. 8. It can be noted that in Case 40-Blk-HiLo, major transmission investments happen in 2020, 2025, and 2030. However, for Case 20-Blk-Avg, the major investments are in 2025, 2029, and 2030. Case 40-Blk-HiLo produces an earlier TEP than Case 20-Blk-Avg. (The reason for the extensive transmission investment in these years is due to the concentrated expansion of generation in these years. Since the power generated by the newly built wind generation needs to be transmitted interregionally, it is more economic that the generation and transmission expansion remains synchronous. Fig. 9 shows the annual generation expansion capacity. It verifies that the years with large generation expansions coincide with those of large transmission expansion for both cases.) 


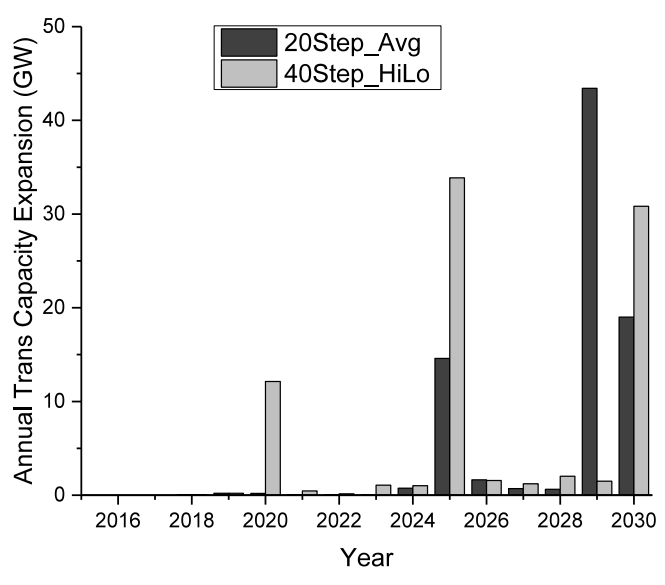

Fig. 8. Transmission capacity expansion of Case 20-Blk-Avg and Case 40-Blk-HiLo.

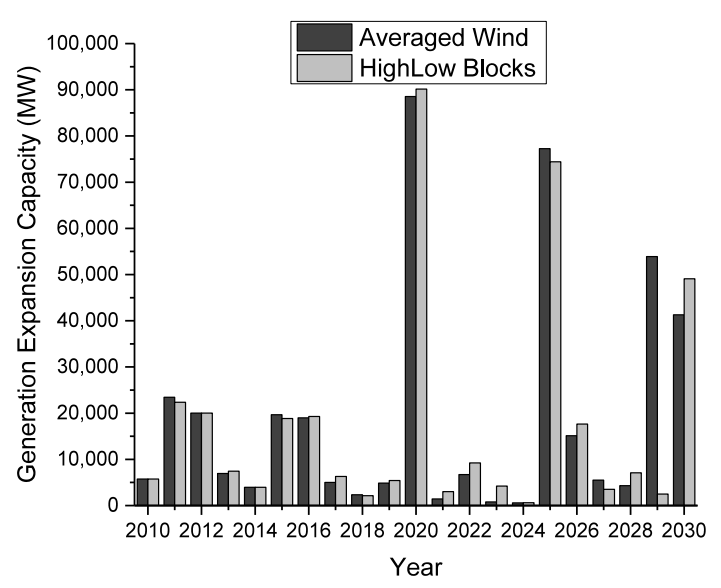

Fig. 9. Annual generation expansion capacity of Case 20-Blk-Avg and Case 40-Blk-HiLo.

Fig. 10 shows the expanded transmission capacity by each interface in 2030. In Case 20-Blk-Avg, nine transmission interfaces' capacities are expanded, while 14 interfaces are expanded in Case 40-Blk-HiLo. It shows that the transmission expansion is more evenly distributed in Case 40-Blk-HiLo.

In Case 20-Blk-Avg, the three interfaces that have major expansion are SPP N to MISO MO-IL, MISO MO-IL to MISO IN, and MISO IN to PJM ROR, which forms a wind power delivery path from SPP N to MISO MO-IL, MISO IN, and then to PJM ROR. In contrast, Case 40-Blk-HiLo reduces the expansion in SPP N to MISO MO-IL and MISO IN to PJM ROR and increase expansion in MISO MO-IL to MISO W and other interfaces. In addition to the increase of total transmission investment, expansion of transmission is more dispersed in terms of time and space after incorporating more wind levels.

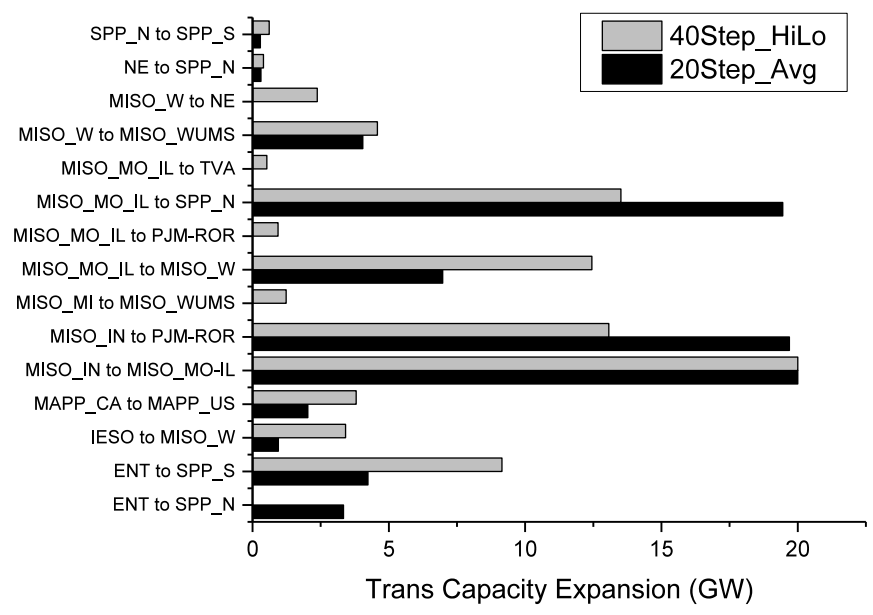

Fig. 10. Expanded transmission capacity in 2030.

The maximum and minimum flows of interfaces in the year 2030 of the two cases are shown Fig. 11 (Case 20-Blk-Avg) and Fig. 12 (Case 40-Blk-HiLo). It shows that the interregional flow of the two cases' 
planning results are similar: power is transmitted from rich wind regions (MISO W, SPP N and MISO MO-IL) to load center regions (PJM, TVA and ENT). However, an obvious difference is that the maximum flow in the interface between MISO MO-IL and SPP N is much larger for Case 20-Blk-Avg than that of Case 40-Blk-HiLo.

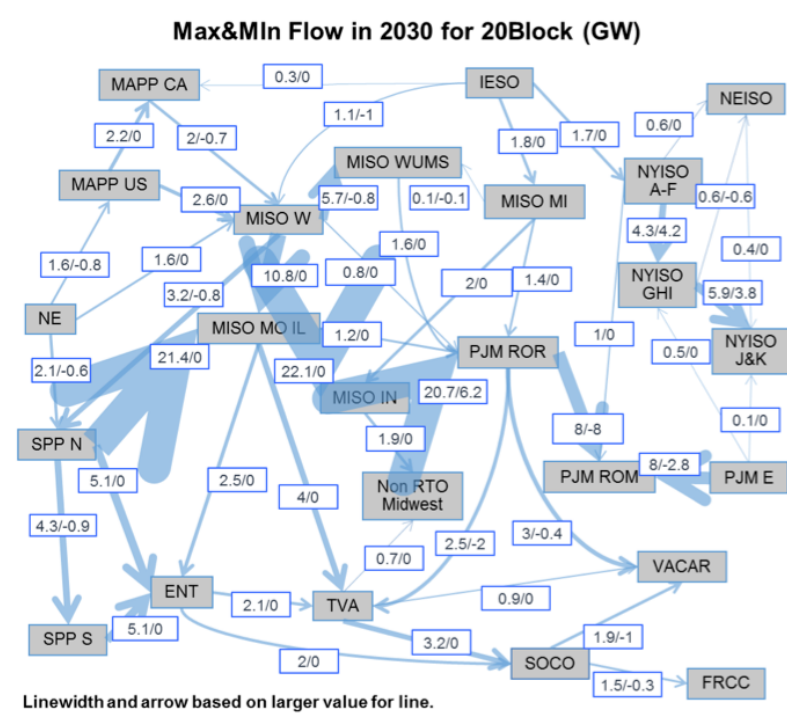

Fig. 11. The maximum and minimum flow for Case 20-Blk-Avg.

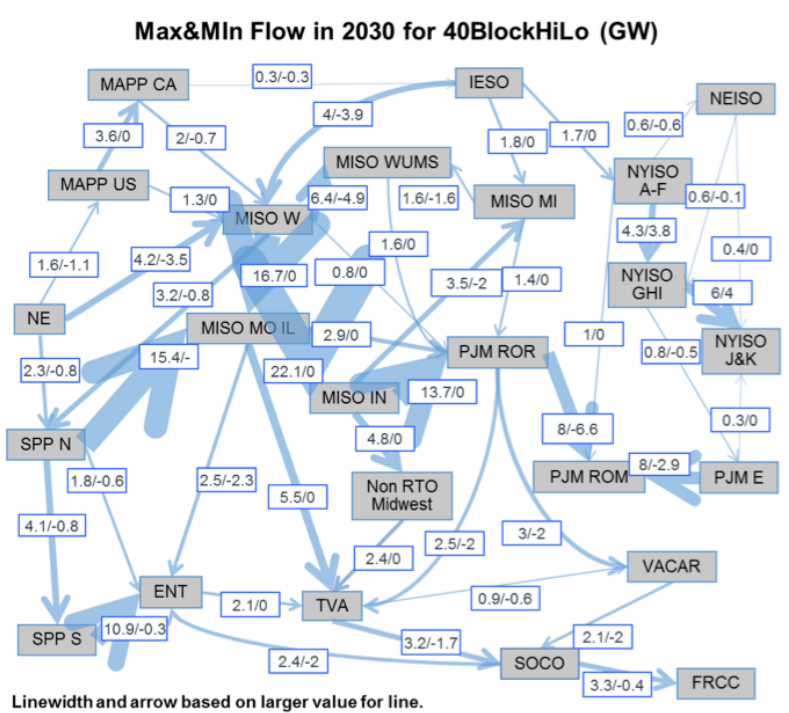

Fig. 12. Maximum and minimum flow for Case 40-Blk-HiLo.

The interface average flows in the year 2030 are shown in Fig. 13 (Case 20-Blk-Avg) and Fig. 14 (Case 40-Blk-HiLo). It can be seen that the averaged flows in Case 40-Blk-HiLo are generally smaller than those in Case 20-Blk-Avg. Also, the major wind power transmission corridor SPP N $\rightarrow$ MISO MO$\mathrm{IL} \rightarrow$ MISO IN $\rightarrow$ PJM ROR in the Case 20-Blk-Avg case reduces its capacity, while a minor corridor MISO W $\rightarrow$ MISO MO-IL $\rightarrow$ MISO IN $\rightarrow$ PJM ROR increases its capacity in Case 40-Blk-HiLo. This result shows that the model using smoother wind data will result to excessive dependence on the wind resources in certain regions and neglect their unreliability during low-wind scenarios, in which system operation cost may increase substantially. Considering both the high- and low-wind scenarios will produce better expansion planning, avoiding excessive investment in just a few interfaces and regions.

In addition, by comparing Fig. 13 and Fig. 14, it can be noted that the transmission planning for those regions located in the edge areas will be more robust in Case 40-Blk-HiLo. For example, the average power flow values for the interface ENT-SPP $S$ in the two cases are very similar. However, as shown in Fig. 10, the expanded capacity is 4,558 MW in Case 20-Blk-Avg, compared with 9,174 MW in Case 40-Blk-HiLo. This indicates that the expansion result in Case 20-Blk-Avg cannot fully provide the transmission capacity required in actual operation, since using the average wind power cannot reflect the transmission capability requirements under high-wind scenarios. A similar phenomenon can be found in interfaces MISO W $\rightarrow$ NE and MAPP US $\rightarrow$ MAPP CA. In these interfaces, the expanded capacities are higher in Case 40-Blk-HiLo than Case 20-Blk-Avg, but these expanded interfaces are actually capable of transmitting only a smaller amount of annual energy than Case 20-Blk-Avg. 


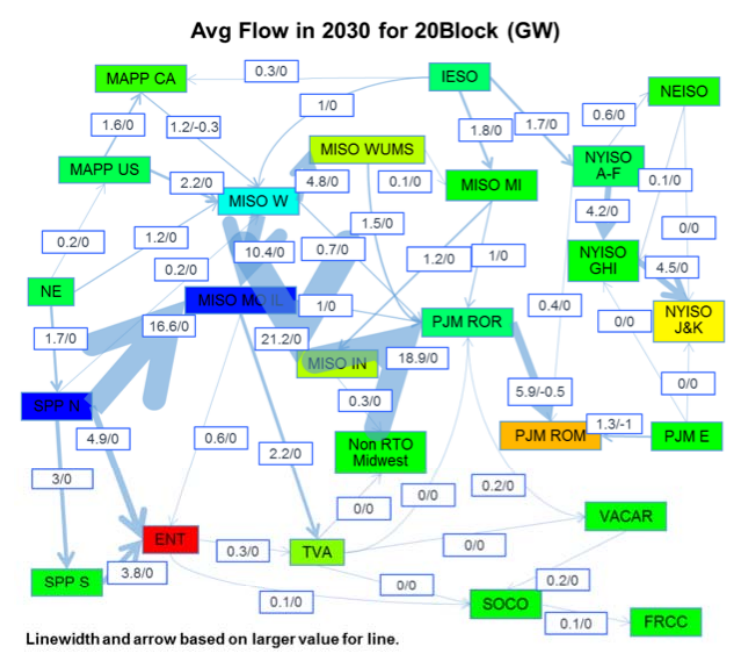

Fig. 13. Average energy flow in 2030

(Case 20-Blk-Avg).

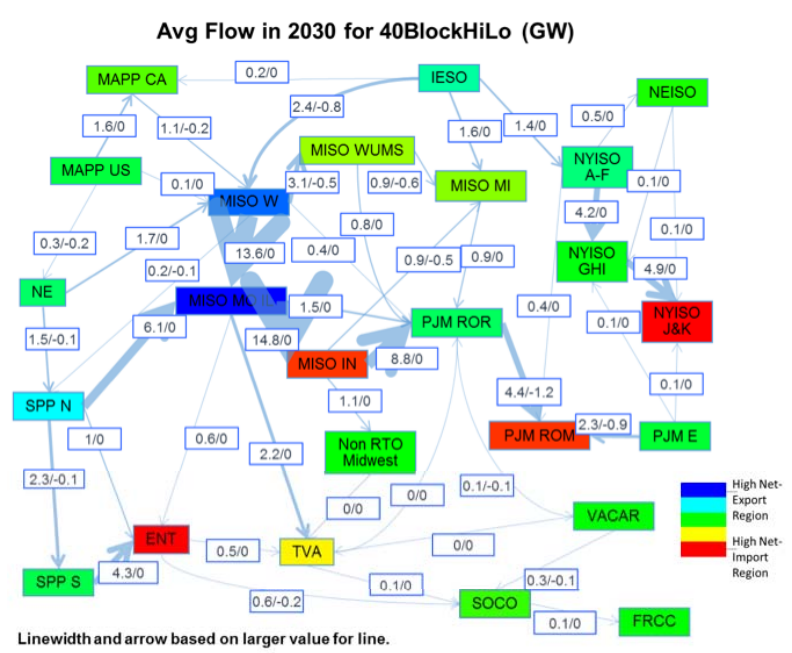

Fig. 14. Average energy flow in 2030 (Case 40-Blk-HiLo).

The cost breakdown for the two cases is shown in Table 5. Besides transmission expansion costs, it shows that the generation cost and the emission cost in Case 40-Blk-HiLo are higher than those in Case 20-Blk-Avg. That is due to that Case 40-Blk-HiLo preserves high market price scenarios when wind power output is low. The generation expansion cost for Case 40-Blk-HiLo is smaller than that of Case 20-Blk-Avg, which is primarily caused by reduced wind generation expansion.

Table 5. Cost breakdown of Case 20-Blk-Avg and Case 40-Blk-HiLo

\begin{tabular}{lcc}
\hline \multicolumn{1}{c}{ Cost } & Case 20-Blk-Avg (billion \$) & Case 40-Blk-HiLo (billion \$) \\
\hline Gen Production Cost (NPV) & 1,253 & 1,266 \\
Emission Cost (NPV) & 522 & 526 \\
Gen Build Cost (NPV) & 649 & 595 \\
Trans Build Cost (NPV) & 20 & 26 \\
Wheeling Charges on Interfaces(NPV) & 15 & 16 \\
\hline
\end{tabular}

Fig. 15 shows the generation expansion capacity by region in 2030. Fig. 16 and Fig. 17 show the annual generation capacity by fuel types of two cases in SPP N. It can be seen that Case 40-Blk-HiLo substantially decreases wind generation expansion in SPP N. 


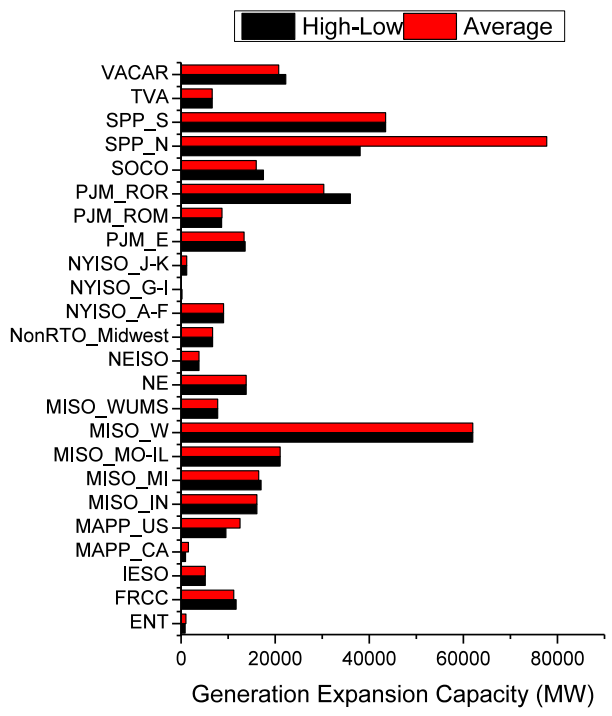

Fig. 15. Expanded generation capacity by regions in 2030 (Case 20-Blk-Avg and Case 40-Blk-HiLo).

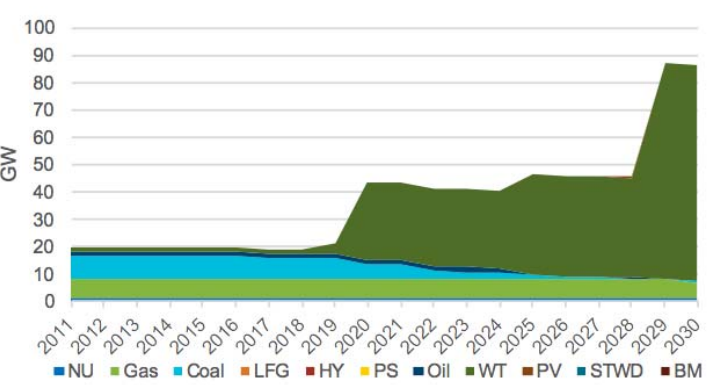

Fig. 16. Generation capacity by fuel in SPP $N$ (20 Blocks Avg)

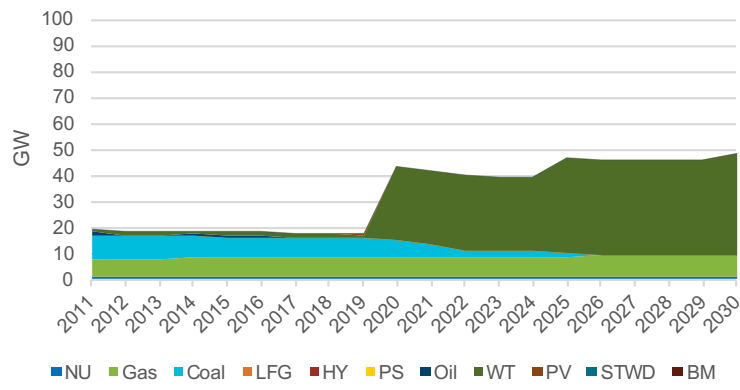

Fig. 17. Generation capacity by fuel in SPP N (40 Blocks HiLo)

\subsection{IMPACT OF WIND SPATIAL DIVERSITY ON LT PLANNING}

Case 40-Blk-Sync considers the diversity of wind and load data across regions in expansion optimization. Table 6 shows a summary of the expansion result of Case 40-Blk-Sync and Case 20-Blk-Avg, as well as Case 40-Blk-HiLo.

In Table 6, it can be seen that the planning results of Case 40-Blk-Sync is between that of Case 20-BlkAvg and Case 40-Blk-HiLo. Since Case 20-Blk-Avg assumes that wind output is the average value during a period, it overestimates the capacity value of wind power and underbuilds transmission capacity. Case 40-Blk-HiLo improves the result by considering more wind scenarios, but it underestimates the capacity value of wind power since it assumes the wind power of all regions is at the high or low half simultaneously. Fig. 18 shows the interregional energy flow in 2030 for Case 40-Blk-Sync. It shows that the amount of energy interchange of Case 40-Blk-Sync is between that of Case 20-Blk-Avg (Fig. 13) and Case 40-Blk-HiLo (Fig. 14). 
Table 6. Summary of the expansion results of Case 40-Blk-Sync

\begin{tabular}{lccc}
\hline \multicolumn{1}{c}{ Expansion results } & Case 20-Blk-Avg & Case 40-Blk-HiLo & Case 40-Blk-Sync \\
\hline Wind Candidates Built Capacity (GW) $^{a}$ & 262 & 218 & 223 \\
Gen Built Capacity (GW) & 407 & 373 & 381 \\
Total Wind Capacity (GW) & 304 & 260 & 265 \\
Wind Generation in 2030 (TWh) & 917 & 768 & 783 \\
Gen Build Cost (NPV) (billion \$) & 649 & 595 & 603 \\
Trans Build Cost (NPV) (billion \$) & 20.2 & 26.1 & 22.1 \\
Emission in 2030 (million ton) & 305 & 365 & 358 \\
Fuel Offtake in 2030 (Quads) & 17.1 & 18.2 & 18.1 \\
\hline
\end{tabular}

${ }^{a}$ Not including wind power that has already been decided to build.

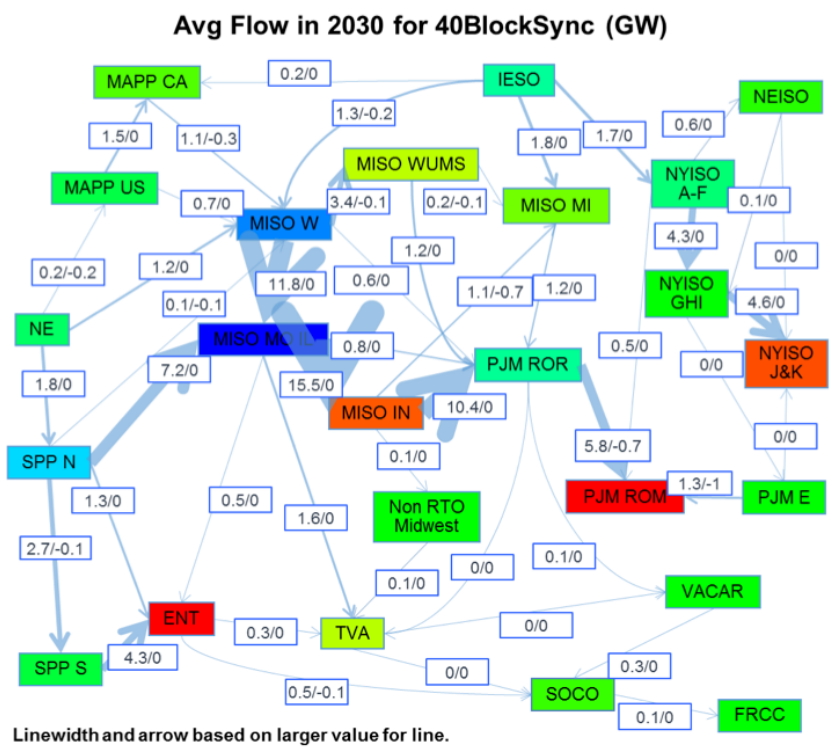

Fig. 18. Average energy flow in the year 2030 (Case 40-Blk-Sync).

\subsection{IMPACT OF MORE DETAILED REPRESENTATION OF WIND POWER VARIATION ON EXPANSION RESULTS}

In Sections 2.3 and 2.4, we showed that Case 40-Blk-HiLo can represent the some temporal diversity and Case 40-Blk-Sync can consider the spatial diversity between demand, wind, and solar data. In this section, we consider using more blocks to represent more scenarios of wind power output. Each block in the Case 20-Blk-Avg case is split into four blocks (the value of each block representing different levels of wind power output: $0-25 \%, 25-50 \%, 50-75 \%$, and 75-100\%) and eight blocks (the value of each block representing $0-12.5 \%, 12.5-25 \%, 25-37.5 \%, 37.5-50 \%, 50-62.5 \%, 62.5-75 \%, 75-87.5 \%$, and $87.5-$ $100 \%)$. Then the demand and solar data are synchronized with the wind data and form their own 80 aggregated blocks for LT planning. Table 7 presents the costs information on the expansion results for the five cases. 
Table 7. Summary of expansion results of Case 80-Blk-Sync and Case 160-Blk-Sync

\begin{tabular}{lccccc}
\hline \multicolumn{1}{c}{ Expansion results } & $\begin{array}{c}\text { Case 20- } \\
\text { Blk-Avg }\end{array}$ & $\begin{array}{c}\text { Case 40- } \\
\text { Blk-HiLo }\end{array}$ & $\begin{array}{c}\text { Case 40- } \\
\text { Blk-Sync }\end{array}$ & $\begin{array}{c}\text { Case 80- } \\
\text { Blk-Sync }\end{array}$ & $\begin{array}{c}\text { Case 160- } \\
\text { Blk-Sync }\end{array}$ \\
\hline Wind Candidates Built Capacity (GW) $^{a}$ & 262 & 218 & 223 & 221 & 218 \\
Gen Built Capacity (GW) & 407 & 373 & 381 & 380 & 378 \\
Total Wind Capacity (GW) & 304 & 260 & 265 & 263 & 260 \\
Wind Generation in 2030 (TWh) & 917 & 768 & 783 & 776 & 766 \\
Gen Build Cost (NPV) (billion \$) & 649 & 595 & 603 & 601 & 598 \\
Trans Build Cost (NPV) (billion \$) & 20.2 & 26.1 & 22.1 & 22.5 & 25.0 \\
Emission in 2030 (million tons) & 305 & 365 & 358 & 362 & 368 \\
Fuel Offtake 2030 (million GBTU) & 17.1 & 18.2 & 18.1 & 18.1 & 18.2 \\
\hline
\end{tabular}

${ }^{a}$ Not including wind power that has already been decided to build.

Table 7 shows that Case 80-Blk-Sync and Case 160-Blk-Sync add less wind than 40-Blk-Sync but more transmission. This is because higher wind peak generation in top blocks needs more transmission capacity to export power. In the meantime, lower wind blocks reduce wind power's capacity value, thereby reducing wind power expansion. Fig. 19 shows the transmission expansion results of the four cases. It can be seen that Case 80-Blk-Sync and Case 160-Blk-Sync have larger transmission expansion than Case 40-Blk-Sync. It indicates that using more detailed wind scenarios will make the transmission expansion more dispersed in space. Particularly, compared with Case 20-Blk-Avg, Case 40-Blk-Sync, Case 80-BlkSync, and Case 160-Blk-Sync have smaller transmission expansion on the interface MISO IN to PJM ROR.

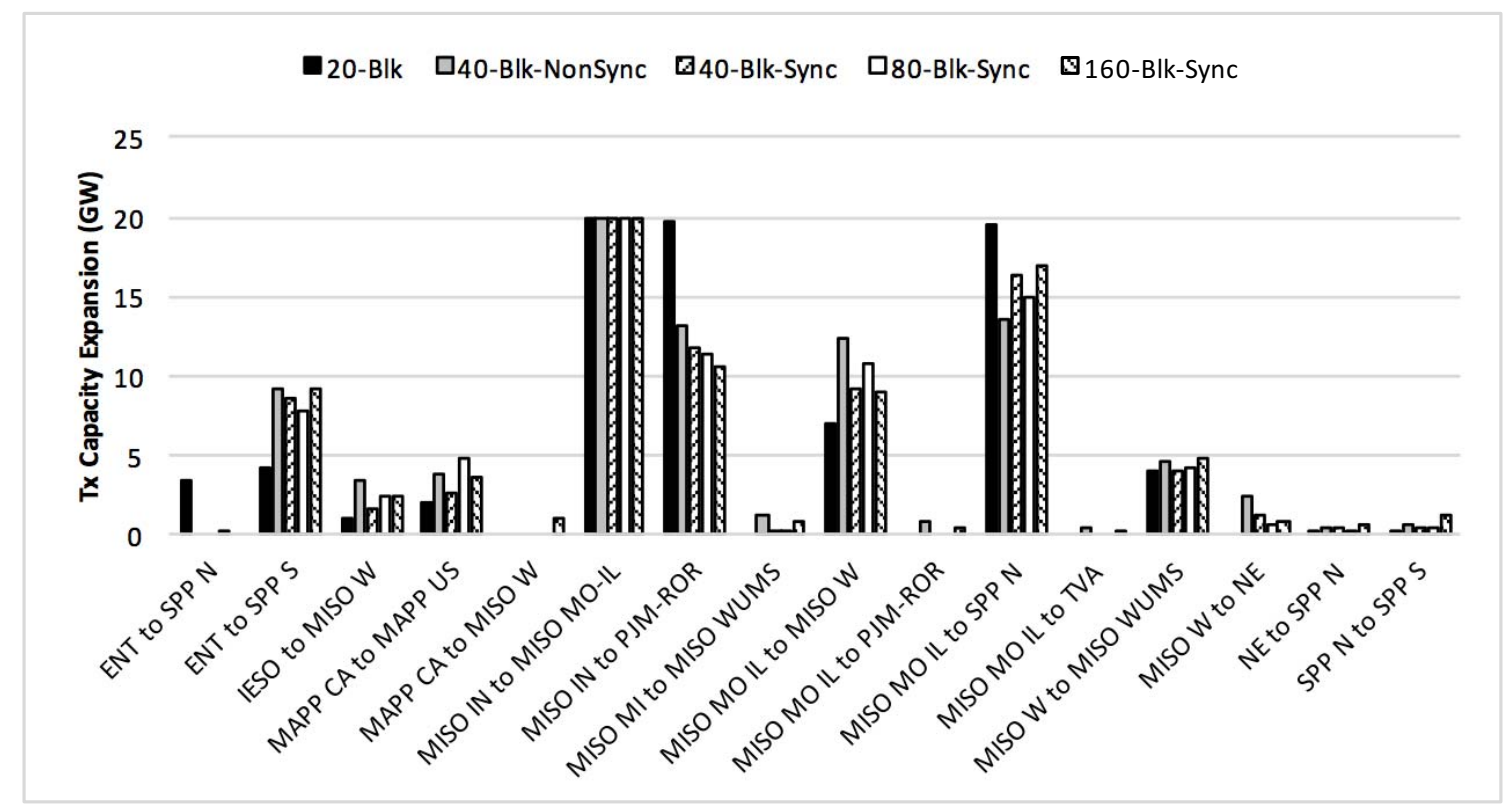

Fig. 19. Transmission expansion in 20 Blocks, Case 40-Blk-HiLo, Case 40-Blk-Sync, and 80 Blocks sync.

Table 8 shows the expansion of gas and wind generation capacity in PJM ROR and SPP N. Fig. 20 and Fig. 21 show the energy flow in 2030 for Case 20-Blk-Avg and Case 160-Blk-Sync, respectively. It shows that in Case 20-Blk-Avg, a large proportion of import energy to PJM ROR comes from the wind in SPP N. When more wind diversity is considered, PJM ROR relies more on its own cheap gas generation (Case 40-Blk-HiLo and 40/80/160-Blk-Sync). For comparison, Fig. 22 shows the annual energy flow of the not-co-optimized case (which optimizes generation and transmission expansion separately). It can be 
seen that this expansion result chooses to expand the interface between MISO W and PJM ROR. In fact, expansion of this interface requires high investment and causes higher operation costs, making the expansion plan uneconomic.

For comparison, Fig. 22 shows the annual energy flow of the not-co-optimized case (which optimizes transmission and generation expansion sequentially [46]). It can be seen that the not-co-optimized solution chooses to expand the interface between MISO W and PJM ROR. In fact, expansion of this interface requires high investment, making the whole expansion plan uneconomic. On the contrary, the co-optimized case is able to leverage multiple regions with good wind resources (e.g., MISO W and SPP $\mathrm{N}$ ) and transfer this energy through an optimal path to regions with high load and low wind (e.g., PJM).

Table 8. Expansion of gas and wind generation in PJM ROR and SPP N for the five cases

\begin{tabular}{lccccc}
\hline \multicolumn{1}{c}{ Expansion Result } & $\begin{array}{c}\text { Case 20-Blk- } \\
\text { Avg }\end{array}$ & $\begin{array}{c}\text { Case 40-Blk- } \\
\text { HiLo }\end{array}$ & $\begin{array}{c}\text { Case 40-Blk- } \\
\text { Sync }\end{array}$ & $\begin{array}{c}\text { Case 80-Blk- } \\
\text { Sync }\end{array}$ & $\begin{array}{c}\text { Case 160- } \\
\text { Blk-Sync }\end{array}$ \\
\hline $\begin{array}{l}\text { PJM ROR gas combined- } \\
\text { circle expansion (GW) }\end{array}$ & 6 & 12 & 15.5 & 16 & 17.5 \\
$\begin{array}{l}\text { SPP N wind expansion } \\
\text { (GW) }\end{array}$ & 76.8 & 37.4 & 41.0 & 37.4 & 37.4 \\
PJM ROR net interchange & $\begin{array}{c}153 \mathrm{TWh} \\
\text { Import }\end{array}$ & $\begin{array}{c}82 \mathrm{TWh} \\
\text { Import }\end{array}$ & $\begin{array}{c}78 \mathrm{TWh} \\
\text { Import }\end{array}$ & $\begin{array}{c}69 \mathrm{TWh} \\
\text { Import }\end{array}$ & $\begin{array}{c}61 \mathrm{TWh} \\
\text { Import }\end{array}$ \\
\hline
\end{tabular}

${ }^{a}$ Not including wind power that has already been decided to build.

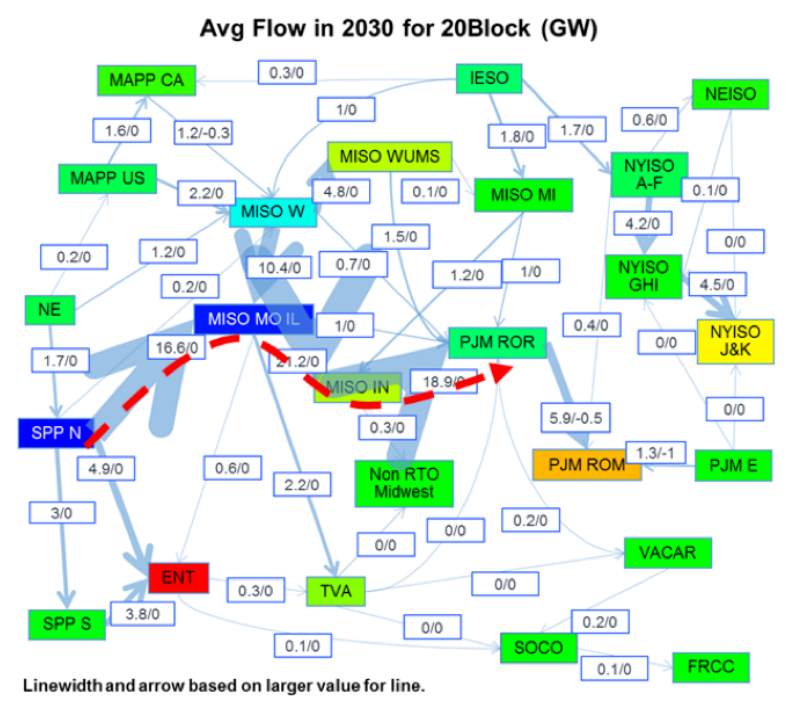

Fig. 20. The annual energy flow in 2030

(Case 20-Blk-Avg) (Linewidth is proportional to interface annual energy flow).

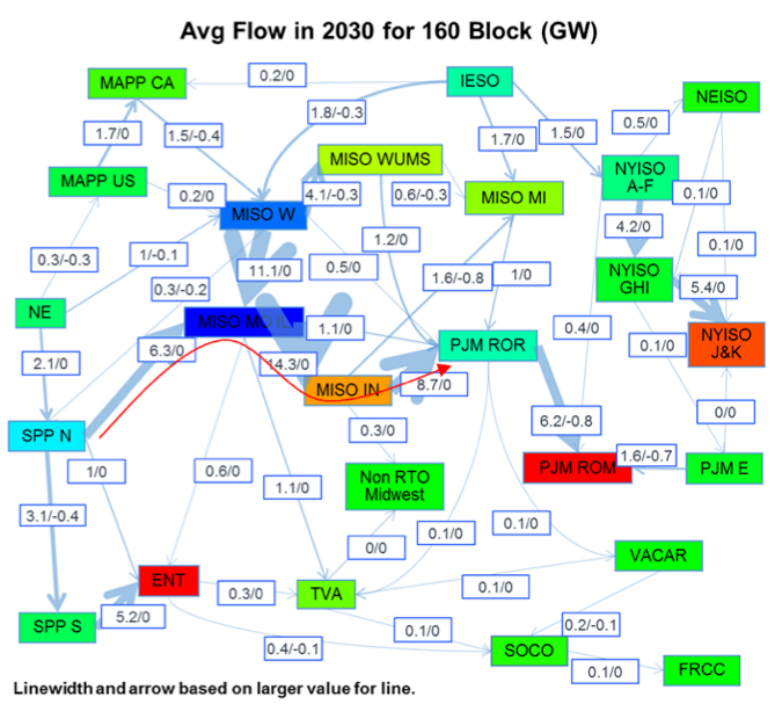

Fig. 21. The annual energy flow in 2030 (Case 160-Blk-Sync) (Linewidth is proportional to interface annual energy flow). 


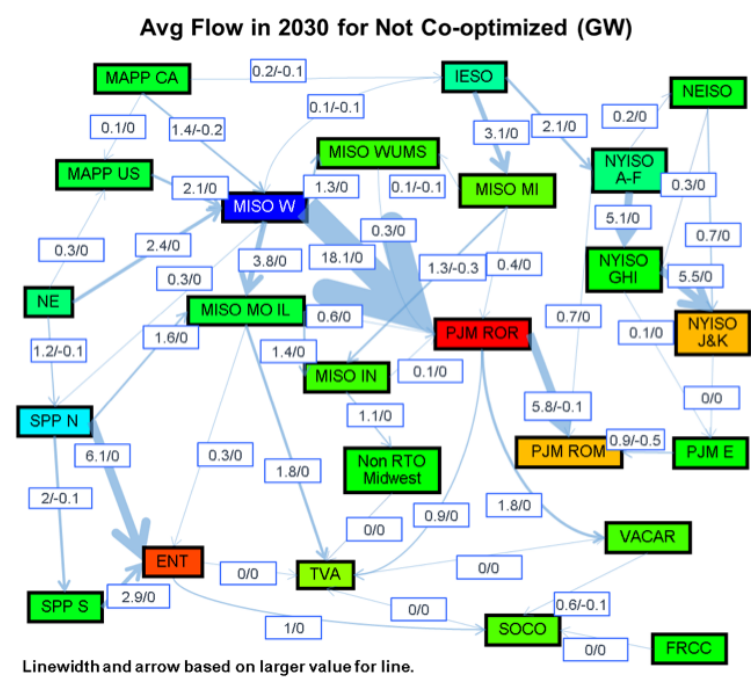

Fig. 22. Annual energy flow of the not-co-optimized case (Linewidth is proportional to interface annual energy flow).

\subsection{ST AND LT SIMULATION RESULTS COMPARISON}

In order to quantify the accuracy improvement through the proposed scenario creation method, the LT expansion result is compared with the ST simulation result for each case. The LT simulation applies optimized power flow calculations based on the blocks generated in the expansion planning model, while ST simulation uses unit commitment and economic dispatch based on the chronological hourly data. In Table 9, the LT and ST comparison shows that there are gaps between the ST and LT results. This is caused by LT expansion using the aggregated blocks that omit some information in the hourly data, which is too detailed to be considered in LT planning. In addition, it shows that Case 160-Blk-Sync has the smallest gap between LT and ST simulations, indicating that Case 160-Blk-Sync has the most accurate operation simulation incorporated in its LT planning model among these cases. Therefore, the expansion co-optimization result obtained in Case 160-Blk-Sync is more reasonable (but at the expense of execution time). These results show that modelling more blocks of wind power and synchronizing other data of all regions can make the production simulation more accurate in LT expansion planning.

Table 9. ST and LT simulation results in 2030 for the five cases

\begin{tabular}{lcccccc}
\hline \multicolumn{1}{c}{ Results } & LT/ST & $\begin{array}{c}\text { Case 20-Blk- } \\
\text { Avg }\end{array}$ & $\begin{array}{c}\text { Case 40-Blk- } \\
\text { NonSync }\end{array}$ & $\begin{array}{c}\text { Case 40-Blk- } \\
\text { Sync }\end{array}$ & $\begin{array}{c}\text { Case 80-Blk- } \\
\text { Sync }\end{array}$ & $\begin{array}{c}\text { Case 160- } \\
\text { Blk-Sync }\end{array}$ \\
\hline $\begin{array}{l}\text { Generation } \\
\text { cost (NPV } \\
\text { billion \$) }\end{array}$ & LT & 47.9 & 55.0 & 54.4 & 54.9 & 55.2 \\
\hline $\begin{array}{l}\text { Emission } \\
\text { cost (NPV }\end{array}$ & LT & 60.3 & 61.9 & 60.0 & 59.7 & 59.5 \\
billion \$) & ST & 42.8 & 51.0 & 50.0 & 50.5 & 51.3 \\
\hline LT-ST gap & 50.9 & 52.6 & 52.5 & 53.1 & 53.2 \\
\hline Computation time & & $18.36 \%$ & $7.48 \%$ & $7.13 \%$ & $6.57 \%$ & $5.52 \%$ \\
\hline
\end{tabular}


In addition, it can be noted that there are always gaps between the ST and LT results. This is because LT expansion uses the aggregated time blocks, which omit some information in the hourly data. Also, as the number of blocks increases, the computation time increases nonlinearly. Therefore, a balance between the number of blocks and computation efforts should be considered.

\subsection{SUMMARY}

In this report, generation and transmission expansion is co-optimized using the proposed MIP model, which can be solved robustly using MIP solvers to obtain the global optimal solution. A block creation method is proposed to represent wind and load diversities of different regions effectively, thus modelling the interchange of energy between regions more accurately. This block creation method can efficiently incorporate uncertainties and operation details into the MIP model to obtain a better expansion plan. The co-optimization model and the block creation method are verified by comparing the LT and ST simulation results of the US EI system. Additional findings on implementing the proposed framework to the case study are as follows.

1) Compared with separated optimization, the co-optimized model is able to better leverage wind resources and find a cost-effective path to transmit energy to regions with high load and low wind. The MIP formulation features a systematic consideration of generation and transmission expansion resources.

2) Incorporating the diversity of wind speed by using more blocks will decrease the wind expansion capacity and make transmission expansion more dispersed in space. In addition, detailed wind blocks will reveal that it may be less economic to expand transmission networks to transmit a large amount of wind power over a long distance in the EI system. 



\section{PRELIMINARY EXPERIMENTS ON HPC-BASED PARALLEL SIMULATIONS OF POWER SYSTEM OPERATION AND PLANNING}

With the expansion in the interconnected power grids, the size and complexity of operation and planning simulations have increased significantly. A simulation task for an actual system can easily exceed the computing capability of a desktop, so there is a growing need to leverage HPC resources for large-scale and LT power system simulations. The computation time of power system simulation depends on many factors, including model features and complexity, the horizon of simulation/optimization, solving algorithms, hardware, and parallel computation techniques, etc. Details of the influence factors include the following.

- Model complexity measures the scale of the problem to be solved. Indicators of model complexity include the number of decision variables and state variables, the number of constraints, parameters sparsity, and whether it contains stochastic variables and multi-scenarios or not. The length of the studying horizon is a common impact factor on model complexity (such as in LT expansion optimization, ST market simulation, and dynamic simulation). The horizon length and computation complexity may be linearly or nonlinearly correlated, depending on whether the problem is timecoupled or not.

- Problem features could be a more decisive factor of computation complexity compared with complexity. The computation burden may vary greatly with different problem types, such as linear, nonlinear, and differential equations and the inclusion of integer variables. Other features of the problems, such as the percentage of binding constraints and the physical coupling of the system, could also be important influential factors on computation.

- Hardware performance, such as the clock rate, memory r/w speed, hard disk speed, and the number of cores and threads, could decide the efficiency of executing programs.

- Parallel computation techniques facilitate splitting one simulation task into several sub-tasks that can be executed simultaneously. If they are properly applied, parallel computation techniques may significantly reduce computation time.

This section mainly focuses on parallel computation techniques in LT expansion optimization and ST simulations. The impact of model complexity and hardware on the computation time is studied as well. This section is organized as follows. Section 3.1 studies the relation between the model complexity (the number of blocks) and the computation time under the expansion planning background. Section 3.2 investigates the computation time and efficiency when applying two parallel computation techniques: splitting by chronology (for ST simulation) and splitting by sample (for LT planning). Section 3.3 tests the impact of hardware on computation efficiency.

\subsection{IMPACT OF MODEL COMPLEXITY ON COMPUTATION TIME IN LT PLANNING}

The software version is the same for all testing cases in this report, as presented in Table 10. Table 11 shows the hardware adopted in the studies described in Sections 3.1 and 3.2. 
Table 10. Information on software in the test study

\begin{tabular}{ll}
\hline Software and optimization solver & Parameters \\
\hline Software version & PLEXOS ${ }^{\circledR} 7.100$ R01 x64 Edition \\
PLEXOS $®$ Engine version & 7.100 R01 x64 \\
Optimization solver and version & Xpress-MIP 26.01.04 \\
\hline
\end{tabular}

Table 11. Hardware information of the 6-core machine

\begin{tabular}{ll}
\hline Hardware & Parameters \\
\hline CPU & Intel Xeon E5-1660.0 3.30 GHz \\
Number of cores & 6 \\
Number of threads (virtual cores) & 12 \\
Memory & $64 \mathrm{~GB}$ \\
Operation system & Windows 7 \\
\hline
\end{tabular}

In expansion planning, the load duration curve (LDC) is commonly used to model the load levels over a LT, such as 1 week, 1 month, or 1 year. Table 12 shows the required computation time and resources when a different number of load levels are considered in a three-node model [47] under a 20-year planning horizon.

Table 12. Computation complexity when modelling various number of load levels in a three-node system

\begin{tabular}{lllllll}
\hline $\begin{array}{l}\text { Number of load } \\
\text { blocks (levels) } \\
\text { in one year }\end{array}$ & $\begin{array}{l}\text { LDC } \\
\text { slicing } \\
\text { method }\end{array}$ & $\begin{array}{l}\text { \# of } \\
\text { variables }\end{array}$ & $\begin{array}{l}\text { \# of } \\
\text { constraints }\end{array}$ & $\begin{array}{l}\text { \# of non-zero } \\
\text { elements in the } \\
\text { problem }\end{array}$ & $\begin{array}{l}\text { Memory } \\
\text { required }\end{array}$ & $\begin{array}{l}\text { Total } \\
\text { computation } \\
\text { time }\end{array}$ \\
\hline 1568 & $\begin{array}{l}28 \text { blocks } \\
\text { per week }\end{array}$ & $2,909,060$ & $3,176,044$ & $9,232,304$ & $8,063 \mathrm{MB}$ & $39 \mathrm{~min}$ \\
\hline 3136 & $\begin{array}{l}56 \text { blocks } \\
\text { per week }\end{array}$ & $5,817,700$ & $6,351,804$ & $19,010,624$ & $15,909 \mathrm{MB}$ & $2 \mathrm{~h} 28 \mathrm{~min}$ \\
\hline 4380 & $\begin{array}{l}12 \text { blocks } \\
\text { per day }\end{array}$ & $8,608,740$ & $9,399,164$ & $28,840,923$ & $23,335 \mathrm{MB}$ & $4 \mathrm{~h} 14 \mathrm{~min}$ \\
\hline
\end{tabular}

Table 12 shows that the number of variables (columns) and the number of constraints (rows) in the optimization problem increase linearly with the number of load blocks, while computation complexity increases nonlinearly with the problem size. In the third case, the high time resolution $(2 \mathrm{~h})$ in a 20 -year planning problem leads to a long computation time. It is suggested that a compromise should be reached between the load level number and the computation time. As can be seen from the increase of computation time with the number of load levels in Table 12, large system optimization will be more relied on parallel computation techniques and HPC. The next section studies two parallel computation techniques in power system operation simulation and optimization.

\subsection{TESTING OF PRELIMINARY PARALLEL COMPUTATION TECHNIQUES IN POWER SYSTEM SIMULATION}

Two types of parallel computation techniques are studied here: the parallel execution of LT optimization by samples ("Sample Splitting"), and parallel execution of ST simulation by time partition ("Chronology Splitting"). As a preliminary method to consider uncertainties in optimization, the Sample Splitting technique adopts different values of uncertain factors to construct multiple versions of an optimization problem and then solves them in parallel. Different from robust optimization method which produce a 
single solution, this method is useful when the system planner wants to obtain the probabilistic distribution of system planning indices, such as the distribution of investments and production costs rendered by wind and load variations. The Chronology Splitting technique has been used to accelerate ST market simulations through parallel execution. Ancillary techniques for Chronology Splitting, such as time overlap for seamless chronology partition, has been developed to improve the accuracy [47].

\subsubsection{LT Expansion Planning}

In order to study the Sample Splitting technique in LT planning, five cases are developed, as shown in Table 13. The base case (Case LT 1 Sample Non-Split) is same as in Section 3.1. The samples are generated by sampling wind output, which follows a normal distribution. Among the five cases, the first two cases do not apply Sample Splitting, while the rest of the cases apply this parallel computation technique under a different number of samples.

Table 13. Information on test cases for splitting samples in LT expansion

\begin{tabular}{lll}
\hline \multicolumn{1}{c}{ Case } & Number of samples & \multicolumn{1}{c}{ Splitting tech } \\
\hline LT 1 Sample Non-Split & 1 sample & No splitting applied \\
LT 40 Samples Non-Split & 40 samples & No splitting applied \\
LT 40 Samples Split & 40 samples & Splitting samples \\
LT 200 Samples Split & 200 samples & Splitting samples \\
LT 1000 Samples Split & 1000 samples & Splitting samples \\
\hline
\end{tabular}

The computation time for these cases is shown in Table 14. Comparing LT 1 Sample Non-Split and LT 40 Samples Non-Split, both of which use only one thread to process all samples, it can be seen that the computation time increases with the number of samples. The comparison of LT 40 Samples Non-Split and LT 40 Samples Split shows that splitting samples into different thread for parallel execution decreases the computation time tremendously. After applying the splitting sample technique, the computation time increases with the number of samples assigned to each thread.

Table 14. Computation time of LT cases (splitting samples) on the 6-cores 12-thread machine

\begin{tabular}{lccc}
\hline \multicolumn{1}{c}{ Case } & $\begin{array}{c}\text { Number of samples } \\
\text { proceeded by each thread }\end{array}$ & $\begin{array}{c}\text { Number of working } \\
\text { threads }\end{array}$ & Computation time \\
\hline LT1 Sample Non-Split & 1 & 1 & $10 \mathrm{~s}$ \\
LT 40 Samples Non-Split & 40 & 1 & $1 \mathrm{~min} 34 \mathrm{~s}$ \\
LT 40 Samples Split & 4 & 10 & $23 \mathrm{~s}$ \\
LT 200 Samples Split & 20 & 10 & 1 min 48 s \\
LT 1000 Samples Split & 100 & 10 & 12 min 28s \\
\hline
\end{tabular}

\subsubsection{ST Simulation}

In LT expansion optimization, the availability of generation and transmission is time-coupled since the construction or retirement of generation and transmission resources will have an effect on system operation in the rest of the studied horizon. This feature makes LT expansion planning difficult to be split by chronology. On the contrary, in ST simulations, the availability of generation and transmission resource is usually known, so ST simulation is loosely coupled in time over longer periods. For example, the ST operation of this month and the next month is relatively independent as the more decisive factors are the load variation and resource availability in those periods. By splitting the simulation horizon into 
multiple sub-horizons, the Chronology Splitting technique can leverage the computation resources of multi-core machines to greatly increase ST simulation efficiency.

To test Splitting Chronology, two ST simulate cases are created based on the three-node system [47]. The ST simulation utilizes the hourly data over a 5-year horizon to simulate system operation under a market environment. Information on the two ST cases is provided in Table 15. In the case that applies Splitting Chronology (ST 5 Year Split), the horizon is split into half years for ST simulation and stitched together as the final result.

Table 15. Information on test cases for splitting chronology in ST operation simulation

\begin{tabular}{cll}
\hline Case & Horizon & Splitting technology \\
\hline ST 5 Year Non-Split & 5 years & Non-split \\
ST 5 Year Split & 5 years & Split chronology \\
\hline
\end{tabular}

The computation times of the two cases on the 6-core 12-thread machine are presented in Table 16. It shows that the simulation time significantly decreases after applying the chronology partition technique.

Table 16. Computation time of ST cases (splitting chronology) cases on the 6-core 12-thread machine

\begin{tabular}{lccccc}
\hline \multicolumn{1}{c}{ Case } & $\begin{array}{c}\text { Total number of } \\
\text { sub-horizons }\end{array}$ & $\begin{array}{c}\text { Number of } \\
\text { chronology horizon } \\
\text { in each thread }\end{array}$ & $\begin{array}{c}\text { Duration of each } \\
\text { sub-horizon }\end{array}$ & $\begin{array}{c}\text { Number of } \\
\text { working } \\
\text { threads }\end{array}$ & $\begin{array}{c}\text { Computation } \\
\text { time }\end{array}$ \\
\hline ST 5-Year Non-Split & 1 & 1 & 5 year & 1 & 3 min 34 s \\
ST 5-Year Split & 10 & 1 & A half year & 10 & $42 \mathrm{~s}$ \\
\hline
\end{tabular}

\subsection{TESTING OF HARDWARE'S IMPACT}

The same cases in Section 3.2 are applied to a 20-core 40-thread machine, whose hardware information is shown in Table 17. The computation time of the five LT cases on the 20-core 40-thread machine is shown in Table 18

Table 17. Hardware information of the 20-core 40-thread machine

\begin{tabular}{ll}
\hline \multicolumn{1}{c}{ Hardware } & \multicolumn{1}{c}{ Parameter } \\
\hline CPU & Intel Xeon E5-2470.0 V2 2.40 GHz \\
\# of cores & 20 \\
\# of threads & 40 \\
Memory & $128 \mathrm{~GB}$ \\
Operation system & Windows Server 2 Standard \\
\hline
\end{tabular}


Table 18. Computation time of LT cases (splitting samples) on the 20-core 40-thread machine

\begin{tabular}{lccl}
\hline \multicolumn{1}{c}{ Case } & $\begin{array}{c}\text { Number of samples } \\
\text { in each thread }\end{array}$ & $\begin{array}{c}\text { Number of working } \\
\text { threads }\end{array}$ & Computation time \\
\hline LT 1 Sample Non-Split & 1 & 1 & $12 \mathrm{~s}$ \\
LT 40 Samples Non-Split & 40 & 1 & $1 \mathrm{~min} \mathrm{54 \textrm {s }}$ \\
LT 40 Samples Split & 1 & 40 & $25 \mathrm{~s}$ \\
LT 200 Samples Split & 5 & 40 & $45 \mathrm{~s}$ \\
LT 1000 Samples Split & 25 & 40 & 4 min 21 s \\
\hline
\end{tabular}

Table 14 and Table 18 show that the first three cases take more time on the 20-core machine than on the 6-core machine, while the last two cases take less time. Table 19 shows the time consumption of each process of LT 1 Sample Non-Split on two machines. It shows that each process takes more time in the 20core machine than on the 6-core machine. As mentioned before, this case uses only one thread and the clock rates of the two machines are different. The clock rate is $3.3 \mathrm{GHz}$ for the 6-core machine and $2.4 \mathrm{GHz}$ for the 20-core machine. It can be inferred that each core's calculation efficiency is related to the machine clock rate.

Table 19. Computation time of each process in LT expansion optimization on machines with different clock rates

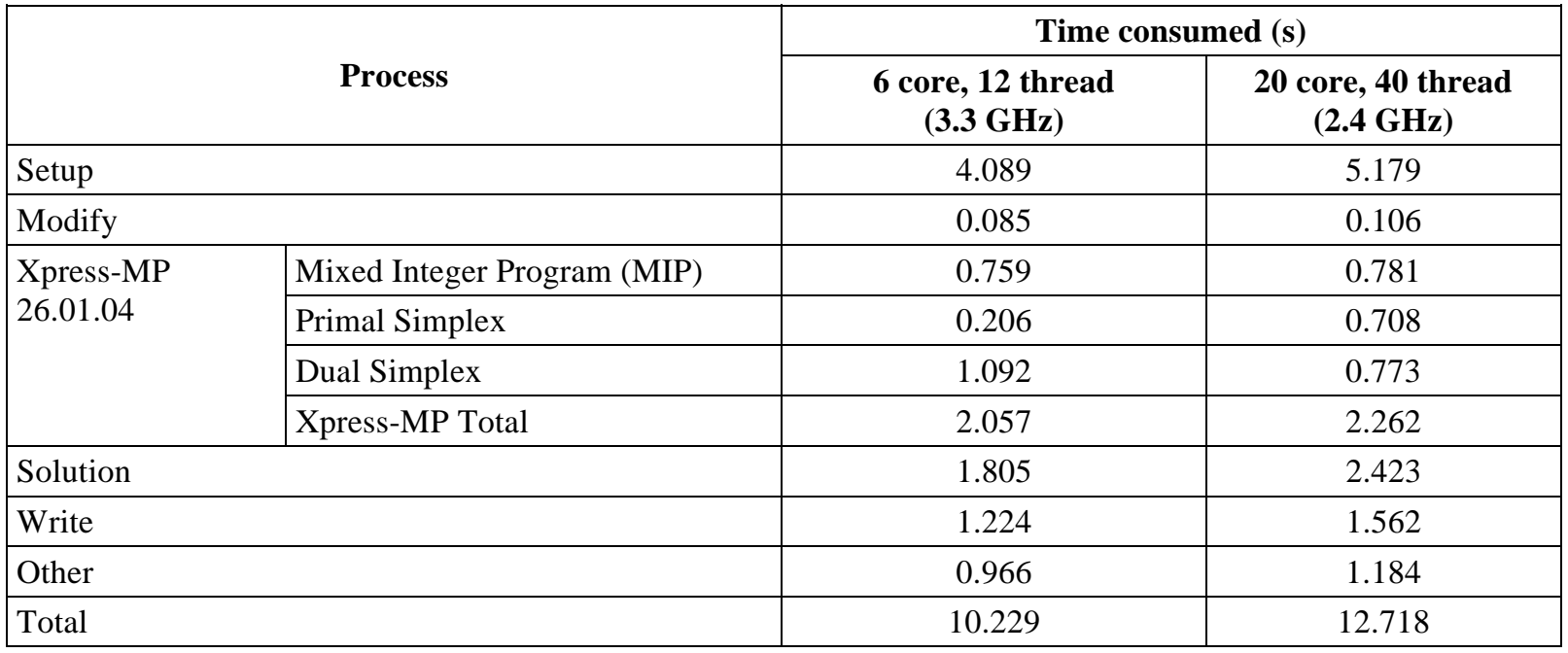

For Case LT 40 Samples Split and Case LT 200 Samples Split, whose numbers of samples are much greater than those of other cases, the advantage of multi-core machines becomes prominent. The computation time decreases tremendously for the 20-core machine compared with the 6-core machine due to more working cores (Table 20). This result shows the potential efficiency increase by combining parallel computation techniques with multi-core machines. 
Table 20. Computation time of ST cases (splitting chronology) on different hardware

\begin{tabular}{llccccc}
\hline Hardware & Case & $\begin{array}{c}\text { Total } \\
\text { number } \\
\text { of sub- } \\
\text { horizons }\end{array}$ & $\begin{array}{c}\text { Number of } \\
\text { chronology } \\
\text { horizon in } \\
\text { each thread }\end{array}$ & $\begin{array}{c}\text { Duration of } \\
\text { each sub- } \\
\text { horizon }\end{array}$ & $\begin{array}{c}\text { Number of } \\
\text { working } \\
\text { threads }\end{array}$ & $\begin{array}{c}\text { Computation } \\
\text { time }\end{array}$ \\
\hline 6 cores & ST 5 Year Non-Split & 1 & 1 & 5 year & 1 & 3 min $34 \mathrm{~s}$ \\
& ST 5 Year Split & 10 & 1 & A half year & 10 & $42 \mathrm{~s}$ \\
20 cores & ST 5 Year Non-Split & 1 & 1 & 5 year & 1 & 4 min $10 \mathrm{~s}$ \\
& ST 5 Year Split & 40 & 1 & 1.5 month & 40 & $24 \mathrm{~s}$ \\
\hline
\end{tabular}

Comparing Table 20 with Table 16, the computation time of Case ST 5-Year Non-Split on the 20-core machine is 4 min $10 \mathrm{~s}$ and is a little longer than that on the 6-core machine, which has a higher clock rate. This result indicates that the computation time of ST simulations in PLEXOS is also related to the clock rate. For Case ST 5-Year Split, in which the splitting chronology technique is applied, the computation time on the 20-core machine is almost half of that on the 6-core machine. It verifies that Chronology Splitting in PLEXOS ST simulations can leverage multi-core and multi-thread resources to boost simulation efficiency.

If there are many simulation tasks, it is necessary to dynamically assign multiple tasks to multiple compute nodes according to task priorities and resource availability. Fig. 23 shows the configuration of a distributed computing network. This computing network consists of a Local Area Network and distributed computation resources, as shown in Table 21. This computing network uses a master-slave architecture to manage computation resources [36]. The master server has centralized data storage and management, and it is able to dynamically distribute computing tasks to slave computing nodes. Upon completion, the solutions in each slave computing nodes are sent back to the master server.

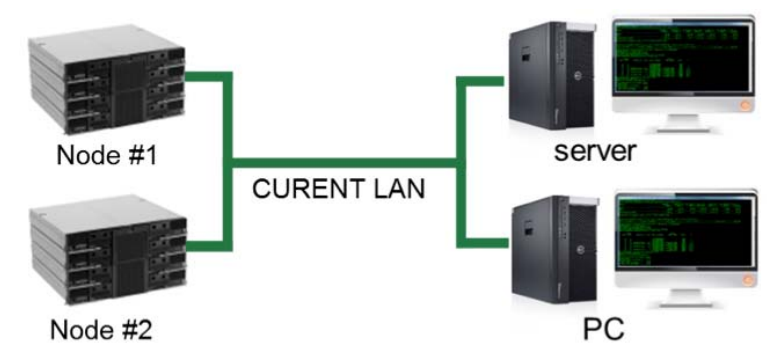

Fig. 23. The PLEXOS® distributed computation network

Table 21. PLEXOS® computation network resources

\begin{tabular}{lcc}
\hline Compute resources & $\begin{array}{c}\text { Number of threads } \\
\text { (virtual cores) }\end{array}$ & Memory \\
\hline Compute Node \#1 & 40 & $130 \mathrm{~GB}$ \\
Compute Node \#2 & 40 & $130 \mathrm{~GB}$ \\
Server \#1 & 12 & $65 \mathrm{~GB}$ \\
PC \#1 & 8 & $8 \mathrm{~GB}$ \\
\hline Total & 100 & $333 \mathrm{~GB}$ \\
\hline
\end{tabular}




\subsection{SUMMARY}

This section tested two preliminary parallel computation techniques based on chronology and sample partition to split the power system simulation task into sub-tasks and then assign these sub-tasks to multiple cores. Testing results show that the simulation time reduced significantly when the simulation task is split into several sub-tasks and assigned to different cores. A summary of this section follows.

1. Splitting Chronology and Sample can leverage multi-core machines to increase simulation efficiency. These techniques can be applied to situations where a problem can be split into multiple subproblems. For example, in ST simulation, time partition can be used to divide the simulation horizon into several sub-horizons for parallel execution. The reduction of computation time confirms the advantage of applying appropriate parallel computation techniques to HPC for power system simulations.

2. When parallel execution is not applied, the CPU clock rate is an influential factor of the computation time. 



\section{CONCLUSIONS AND FUTURE WORK}

In this report, generation and transmission expansion in the EI system is co-optimized using a MIP model based on PLEXOS. The co-optimization problem is solved robustly using commercial MIP solvers. A block creation method is proposed to represent wind and load diversities across regions effectively, thus modelling the interchange of energy between regions of the US EI system more accurately. This block creation method can efficiently incorporate uncertainties and operation details into the MIP model for obtaining a better expansion plan. The co-optimization model and the block creation method are verified by comparing the LT and ST simulation results in the EI system. In addition, two parallel computation techniques for power system simulation are studied on different servers. Conclusions of this study are summarized as follows.

- In LT planning, incorporating high and low wind blocks (temporal diversity) instead of more average blocks will make transmission expansion more dispersed in space and in earlier years. More interregional transmission capacity is needed when incorporating more wind scenarios.

- Incorporating wind power spatial diversity will slightly increase the capacity value of wind power in expansion planning. More blocks and details of wind power modelling in LT planning will represent ST operation more accurately and lead to a better expansion result. The reduced LT-ST gaps show the improvement made by the block creation techniques for LT simulation.

- Splitting and parallel computation techniques show good potential for large power system simulation on HPC resources. Chronology splitting and sample splitting techniques can leverage multi-core machines to accelerate power system simulation.

Future work will focus on the following topics:

- using the EI nodal model to study impact of wind and solar variation on LT co-optimized expansion,

- conducting co-optimization studies to support decisions for each state, and

- considering the probability distributions of wind speed and solar radiation in LT co-optimized expansion. 



\section{REFERENCES}

[1] Y. Gu, J. D. McCalley, and M. Ni, "Coordinating large-scale wind integration and transmission planning," Sustainable Energy, IEEE Transactions on, vol. 3, pp. 652-659, 2012.

[2] G. Latorre, R. D. Cruz, J. M. Areiza, and A. Villegas, "Classification of publications and models on transmission expansion planning," Power Systems, IEEE Transactions on, vol. 18, pp. 938946, 2003.

[3] M. Jenabi, S. M. T. Fatemi Ghomi, and Y. Smeers, "Bi-level game approaches for coordination of generation and transmission expansion planning within a market environment," Power Systems, IEEE Transactions on, vol. 28, pp. 2639-2650, 2013.

[4] R. Hemmati, R.-A. Hooshmand, and A. Khodabakhshian, "Comprehensive review of generation and transmission expansion planning," Generation, Transmission \& Distribution, IET, vol. 7, pp. 955-964, 2013.

[5] A. Lopez, B. Roberts, D. Heimiller, N. Blair, and G. Porro, "US renewable energy technical potentials: a GIS-based analysis," Contract, vol. 303, pp. 275-3000, 2012.

[6] R. Wang, J. Wang, S. You, and S. Wu, "A Novel Transmission Planning Method for Integrating Large-Scale Wind Power," in Power and Energy Engineering Conference (APPEEC), 2012 AsiaPacific, 2012, pp. 1-4.

[7] J. Aghaei, N. Amjady, A. Baharvandi, and M.-A. Akbari, "Generation and Transmission Expansion Planning: MILP-Based Probabilistic Model," Power Systems, IEEE Transactions on, vol. 29, pp. 1592-1601, 2014.

[8] S. Dehghan, N. Amjady, and A. Kazemi, "Two-stage robust generation expansion planning: a mixed integer linear programming model," Power Systems, IEEE Transactions on, vol. 29, pp. 584-597, 2014.

[9] M. C. da Rocha and J. T. Saraiva, "A discrete evolutionary PSO based approach to the multiyear transmission expansion planning problem considering demand uncertainties," International Journal of Electrical Power \& Energy Systems, vol. 45, pp. 427-442, 2013.

[10] V. Hinojosa, N. Galleguillos, and B. Nuques, "A simulated rebounding algorithm applied to the multi-stage security-constrained transmission expansion planning in power systems," International Journal of Electrical Power \& Energy Systems, vol. 47, pp. 168-180, 2013.

[11] G. A. Bakirtzis, P. N. Biskas, and V. Chatziathanasiou, "Generation expansion planning by MILP considering mid-term scheduling decisions," Electric Power Systems Research, vol. 86, pp. 98$112,2012$.

[12] J. H. Zhao, J. Foster, Z. Y. Dong, and K. P. Wong, "Flexible transmission network planning considering distributed generation impacts," Power Systems, IEEE Transactions on, vol. 26, pp. 1434-1443, 2011.

[13] J. Wang, R. Wang, P. Zeng, S. You, Y. Li, and Y. Zhang, "Flexible Transmission Expansion Planning for Integrating Wind Power Based on Wind Power Distribution Characteristics," Journal of Electrical Engineering \& Technology, vol. 10, pp. 709-718, 2015.

[14] J.-B. Park, Y.-M. Park, J.-R. Won, and K. Y. Lee, "An improved genetic algorithm for generation expansion planning," Power Systems, IEEE Transactions on, vol. 15, pp. 916-922, 2000.

[15] M. S. Sepasian, H. Seifi, A. A. Foroud, and A. Hatami, "A multiyear security constrained hybrid generation-transmission expansion planning algorithm including fuel supply costs," Power Systems, IEEE Transactions on, vol. 24, pp. 1609-1618, 2009.

[16] S. Kannan, S. Baskar, J. D. McCalley, and P. Murugan, "Application of NSGA-II algorithm to generation expansion planning," Power Systems, IEEE Transactions on, vol. 24, pp. 454-461, 2009.

[17] P. Murugan, S. Kannan, and S. Baskar, "NSGA-II algorithm for multi-objective generation expansion planning problem," Electric Power Systems Research, vol. 79, pp. 622-628, 2009. 
[18] N. Yang and F. Wen, "A chance constrained programming approach to transmission system expansion planning," Electric Power Systems Research, vol. 75, pp. 171-177, 2005.

[19] J. D. Foster, A. M. Berry, N. Boland, and H. Waterer, "Comparison of mixed-integer programming and genetic algorithm methods for distributed generation planning," Power Systems, IEEE Transactions on, vol. 29, pp. 833-843, 2014.

[20] R. Hemmati, R.-A. Hooshmand, and A. Khodabakhshian, "State-of-the-art of transmission expansion planning: Comprehensive review," Renewable and Sustainable Energy Reviews, vol. 23, pp. 312-319, 2013.

[21] M. Manickavasagam, M. F. Anjos, and W. D. Rosehart, "Sensitivity-based chance-constrained Generation Expansion Planning," Electric Power Systems Research, vol. 127, pp. 32-40, 2015.

[22] B. Gorenstin, N. Campodónico, J. Costa, and M. Pereira, "Power system expansion planning under uncertainty," Power Systems, IEEE Transactions on, vol. 8, pp. 129-136, 1993.

[23] B. Chen, J. Wang, L. Wang, Y. He, and Z. Wang, "Robust optimization for transmission expansion planning: Minimax cost vs. minimax regret," Power Systems, IEEE Transactions on, vol. 29, pp. 3069-3077, 2014.

[24] J. H. Roh, M. Shahidehpour, and L. Wu, "Market-based generation and transmission planning with uncertainties," Power Systems, IEEE Transactions on, vol. 24, pp. 1587-1598, 2009.

[25] S. Dehghan, N. Amjady, and A. J. Conejo, "Reliability-Constrained Robust Power System Expansion Planning," Power Systems, IEEE Transactions on, 2016.

[26] C. L. T. Borges and V. F. Martins, "Multistage expansion planning for active distribution networks under demand and distributed generation uncertainties," International Journal of Electrical Power \& Energy Systems, vol. 36, pp. 107-116, 2012.

[27] D. Delgado and J. Claro, "Transmission network expansion planning under demand uncertainty and risk aversion," International Journal of Electrical Power \& Energy Systems, vol. 44, pp. 696-702, 2013.

[28] H. Yu, C. Chung, and K. Wong, "Robust transmission network expansion planning method with Taguchi's orthogonal array testing," Power Systems, IEEE Transactions on, vol. 26, pp. 15731580, 2011.

[29] P. Maghouli, S. H. Hosseini, M. O. Buygi, and M. Shahidehpour, "A scenario-based multiobjective model for multi-stage transmission expansion planning," Power Systems, IEEE Transactions on, vol. 26, pp. 470-478, 2011.

[30] Y. Feng and S. M. Ryan, "Scenario construction and reduction applied to stochastic power generation expansion planning," Computers \& Operations Research, vol. 40, pp. 9-23, 2013.

[31] G.-R. Kamyab, M. Fotuhi-Firuzabad, and M. Rashidinejad, "A PSO based approach for multistage transmission expansion planning in electricity markets," International Journal of Electrical Power \& Energy Systems, vol. 54, pp. 91-100, 2014.

[32] M. Matar and R. Iravani, "Massively parallel implementation of AC machine models for FPGAbased real-time simulation of electromagnetic transients," Power Delivery, IEEE Transactions on, vol. 26, pp. 830-840, 2011.

[33] P. Aristidou, D. Fabozzi, and T. Van Cutsem, "Dynamic simulation of large-scale power systems using a parallel Schur-complement-based decomposition method," Parallel and Distributed Systems, IEEE Transactions on, vol. 25, pp. 2561-2570, 2014.

[34] H. Ge and S. Asgarpoor, "Parallel Monte Carlo simulation for reliability and cost evaluation of equipment and systems," Electric Power Systems Research, vol. 81, pp. 347-356, 2011.

[35] Charles River Associates, Working Draft of MRN-NEEM Modeling Assumptions and Data Sources for EIPC Capacity Expansion Modeling, 2010.

[36] Energy Exemplar, "PLEXOS Integrated Energy Model," 2014.

[37] S. W. Hadley, Additional EIPC Study Analysis: Interim Report on High Priority Topics, Oak Ridge National Laboratory, 2013.

[38] Y. S. Baek, S. W. Hadley, R. Uria Martinez, G. A. Oladosu, A. M. Smith, F. Li et al., Eastern Interconnection Demand Response Potential, Oak Ridge National Laboratory, 2012. 
[39] S. W. Hadley, D. J. Gotham, and R. L. Luciani, Additional EIPC Study Analysis: Final Report, 2014.

[40] A. Liu, B. Hobbs, J. Ho, J. McCalley, V. Krishnan, M. Shahidehpour et al., "Co-optimization of transmission and other supply resources," prepared for the Eastern Interconnection States' Planning Council, NARUC, 2013.

[41] M. Parashar, J. S. Thorp, and C. E. Seyler, "Continuum modeling of electromechanical dynamics in large-scale power systems," Circuits and Systems I: Regular Papers, IEEE Transactions on, vol. 51, pp. 1848-1858, 2004.

[42] Working Draft of MRN-NEEM Modeling Assumptions and Data Sources for EIPC Capacity Expansion Modeling, 2010.

[43] S. Haffner, A. Monticelli, A. Garcia, J. Mantovani, and R. Romero, "Branch and bound algorithm for transmission system expansion planning using a transportation model," IEE ProceedingsGeneration, Transmission and Distribution, vol. 147, pp. 149-156, 2000.

[44] R. Romero, C. Rocha, M. Mantovani, and J. Mantovani, "Analysis of heuristic algorithms for the transportation model in static and multistage planning in network expansion systems," in Generation, Transmission and Distribution, IEE Proceedings, pp. 521-526, 2003.

[45] S. You, S. Hadley, M. Shankar, and Y. Liu, "Co-optimizing Generation and Transmission Expansion with Wind Power in U.S. Eastern Interconnection," Electric Power systems Research (submitted).

[46] Energy Exemplar, "Co-Optimization of Transmission and Other Resources," ed: National Association of Regulatory Utility Commisioners, 2015.

[47] C. Barrows, M. Hummon, W. B. Jones, and E. T. Hale, Time Domain Partitioning of Electricity Production Cost Simulations, National Renewable Energy Laboratory, 2014. 
